\title{
One dimension photochemical models in global mean conditions in question: application to Titan
}

\author{
M. Dobrijevic ${ }^{a}$ J.C. Loison ${ }^{b}$ V. Hue ${ }^{c}$ T. Cavalié ${ }^{a, d}$

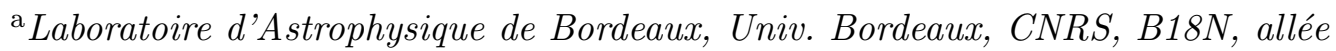 \\ Geoffroy Saint-Hilaire, 33615 Pessac, France \\ ${ }^{\mathrm{b}}$ Institut des Sciences Moléculaires (ISM), CNRS, Univ. Bordeaux, 351 cours de la \\ Libration, 33400, Talence, France \\ ${ }^{\mathrm{c}}$ Southwest Research Institute, San Antonio, TX 78228, United States. \\ ${ }^{\mathrm{d}}$ LESIA, Observatoire de Paris, Université PSL, CNRS, Sorbonne Université, \\ Univ. Paris Diderot, Sorbonne Paris Cité, 5 place Jules Janssen, 92195 Meudon, \\ France
}

13 Number of pages: 31

14 Number of tables: 2

15 Number of figures: 14 
${ }_{16}$ Proposed Running Head:

17 Photochemical model in global mean conditions

18 Please send Editorial Correspondence to:

19

20 Michel Dobrijevic

21 Laboratoire d'Astrophysique de Bordeaux

22 Université de Bordeaux - Bât. B18N

23 Allée Geoffroy Saint-Hilaire

24 CS 50023

2533615 PESSAC CEDEX, France.

${ }_{26}$ Email: michel.dobrijevic@u-bordeaux.fr

27 
Observational data of Titan's atmosphere composition, gathered in particular by the Cassini mission, allow accessing latitudinal, longitudinal and temporal variations of the altitudinal profiles of several species. While 1D models are a powerful tool to gain insight into the chemistry occurring in Titan's atmosphere, they cannot capture the four-dimensional nature of these processes at the same time. However, due to the large extent of the atmosphere and the complexity of chemistry, global 2D and 3D models with the chemical complexity of $1 \mathrm{D}$ models do not exist yet. On the way of developing a 2D photochemical model of Titan, including the very complex chemical schemes used in 1D models and also taking latitudinal variations into account, we developed an ultraviolet radiative transfer model to compute the actinic flux in a 2D geometry. We found that photolysis rates calculated in classical plane-parallel models to derive the rates in mean conditions give results notably different from a model that calculates the mean rates with a 2D geometry. We demonstrate that the introduction of the 2D geometry affects significantly the density profiles on both neutrals and ions, especially on nitriles. As a consequence, we advocate using radiative transfer model in 2D geometry from now on to interpret the wide diversity of observational data about Titan's atmosphere (pending the emergence of 2D models with complex chemical schemes). 
The atmosphere of Titan is a very complex system. Photolysis by solar photons and ionization by Saturn magnetospheric electrons of the two major compounds, molecular nitrogen $\left(\mathrm{N}_{2}\right)$ and methane $\left(\mathrm{CH}_{4}\right)$, are the starting point of a very rich chemistry, enhanced by the surface-atmosphere interaction, influxes from the Enceladus torus (Hartogh et al., 2011; Moreno et al., 2012; Dobrijevic et al., 2014; Lara et al., 2014; Teanby et al., 2018) and interplanetary dust particles (Moses and Poppe, 2017). This efficient chemistry produces aerosols as the end products, which sediment down to the surface, contributing to the radiative budget of the atmosphere and serving as nucleation particles in the lower stratosphere. Modeling Titan's atmosphere is very challenging. In particular, from the chemical point of view, it is necessary to include numerous species and reactions in atmospheric models to study the coupling of neutral, cationic and anionic species composed of various elements like carbon, hydrogen, oxygen, nitrogen and sulfur, throughout the extented atmosphere (see for instance the recent models of Krasnopolsky (2014), Hickson et al. (2014), Mukundan and Bhardwaj (2018), Loison et al. (2019) and Vuitton et al. (2019)). Continuous detections of new molecules, like ethyl cyanide $\left(\mathrm{C}_{2} \mathrm{H}_{5} \mathrm{CN}\right.$, Cordiner et al. (2015)), vinyl cyanide $\left(\mathrm{C}_{2} \mathrm{H}_{3} \mathrm{CN}\right.$, Palmer et al. (2017)), and even isomers like propadiene $\left(\mathrm{CH}_{2} \mathrm{CCH}_{2}\right.$, Lombardo et al. (2019)) and cyclopropenylidene (c- $\mathrm{C}_{3} \mathrm{H}_{2}$, Nixon et al. (2020)), force modelers to constantly improve the chemical scheme they use, rendering codes more and more time consuming. The study of isotopologues (Liang et al., 2007; Mandt et al., 2009; Krasnopolsky, 2016; Loison et al., 2017; Dobrijevic and Loison, 2018) also requires to increase drastically the complexity of the chemical scheme: hundreds of species are necessary to encompass the entire chemical system of Titan's atmosphere in current photochemical models. Two directions can then be taken to model Titan's atmosphere: (1) include limited chemistry to focus on dynamics with 2D or 3D models using some assumptions (for instance: few species, fixed neutral background atmosphere, no cations, no anions, uncoupled chemistry between hydrocarbons and oxygen species, and so on); (2) expand the chemical scheme in 1D models to focus on chemical processes assuming mean conditions with neither latitudinal nor longitudinal transport.

Due to this remarkable chemical complexity, one-dimensional photochemical models are still a powerful tool and used internationally by several teams. However, more and more observational data, in particular those gathered by the Cassini mission, allow accessing latitudinal, longitudinal and temporal variations of the altitudinal profiles of several species (see for instance Vinatier et al. (2010, 2015); Sylvestre et al. (2020)). It is then important to improve these models with the objective to develop 2D photochemical models. As a first step, we advocate to use a 2D geometry to calculate the solar flux available 
for photolysis instead of a plane-parallel approximation in 1D photochemical models.

In the present paper, we compare two types of 1D photochemical models used in mean conditions. In the first model, the photolysis rates are computed by a classic plane-parallel ultraviolet radiative transfer model. In the second model, the ultraviolet radiative transfer model is based on a 2D geometry. In the following, we first present our 1D photochemical model (Section 2) and the two radiative transfer models (Section 3). In Section 4, we compare the photolysis rates calculated in 1D mean conditions and those derived from our $2 \mathrm{D}$ model. We then present the impact of the $2 \mathrm{D}$ photolysis rates on the mole fraction profiles (Section 5) and we conclude in Section 6.

\section{Description of the 1D photochemical model}

The present 1D photochemical model is based on the one presented in Loison et al. (2019) and Dobrijevic et al. (2016). We summarize the main characteristics in the following.

We use a non-uniform altitudinal grid composed of 138 levels from the surface (altitude $\left.z_{0}=0 \mathrm{~km}\right)$ up to the top of the atmosphere $\left(z_{\text {top }}=1500 \mathrm{~km}\right)$ with intervals $\Delta_{z}$ calculated on the basis of value of the atmospheric scale height such as $\Delta_{z}=H_{a} / 5$. Consequently, two consecutive altitudes are separated by $4 \mathrm{~km}$ near the surface and $17 \mathrm{~km}$ near the top of the atmosphere. The temperature profile is the same as in Loison et al. (2015).

The mole fractions of $\mathrm{CH}_{4}, \mathrm{H}_{2}, \mathrm{CO}$ and $\mathrm{Ar}$ are fixed at the lower boundary (surface of Titan): $y_{C H_{4}}=1.48 \times 10^{-2}, y_{H_{2}}=3.0 \times 10^{-3}, y_{C O}=5.1 \times 10^{-5}$ and $y_{A r}=3.39 \times 10^{-5}$ (Loison et al. (2015) and references therein). The mole fraction of $\mathrm{N}_{2}$ is simply given by $y_{N_{2}}=1-\left(y_{\mathrm{CH}_{4}}+y_{\mathrm{H}_{2}}+y_{\mathrm{Ar}}+y_{\mathrm{CO}}\right)$. At the upper boundary, we use an external flux of $\mathrm{O}\left({ }^{3} \mathrm{P}\right)$ and a production of $\mathrm{H}_{2} \mathrm{O}$ from micrometeoritic ablation equal respectively to $1.6 \times 10^{6}$ and $5.2 \times 10^{5} \mathrm{~cm}^{-2} \mathrm{~s}^{-1}$ (values referred to the surface, see Dobrijevic et al. (2014) for details). We assumed a zero flux as an upper boundary condition for all the other species, except for atomic hydrogen $(\mathrm{H})$ and molecular hydrogen $\left(\mathrm{H}_{2}\right)$, which were allowed to escape with velocities following Jean's thermal escape mechanism (this mechanism is negligible for other species). We do not consider sulfur species in the present study since no sulfur species have been detected yet (see Hickson et al. (2014) for model prediction of sulfur-bearing species abundances).

The eddy diffusion coefficient profile is constrained in the present model using argon $(\mathrm{Ar})$ and methane $\left(\mathrm{CH}_{4}\right)$ in the higher atmosphere and water $\left(\mathrm{H}_{2} \mathrm{O}\right)$ and 
hydrogen cyanide (HCN) in the lower atmosphere (see Loison et al. (2019) for details).

The chemical scheme is the same as in Loison et al. (2019) but using recent measurements of the rate constants at low temperatures of $\mathrm{N}\left({ }^{2} \mathrm{D}\right)$ with $\mathrm{CH}_{4}$, $\mathrm{C}_{2} \mathrm{H}_{6}$ and $\mathrm{C}_{3} \mathrm{H}_{8}$ (Nunez-Reyes et al., 2019a) and $\mathrm{N}\left({ }^{2} \mathrm{D}\right.$ ) with $\mathrm{C}_{2} \mathrm{H}_{2}$ (NunezReyes et al., 2019b). Magnetospheric electrons are not taken into account to focus on the effect of photolysis. Our model contains 99 neutral species and 83 ion species (including electron). The chemical scheme has been published in Loison et al. (2019) and modifications are available upon request.

\section{UV radiative transfer model}

\subsection{Actinic flux in a plane-parallel model}

The actinic flux is the quantity of light available to molecules at a particular point in the atmosphere and which, on absorption and ionisation, drives photochemical processes in the atmosphere. This quantity is one of the terms required in the calculation of photolysis rates (photodissociation and photoionisation rates).

In the present study, we compute the actinic flux as a function of altitude in the classic approximation of a plane parallel atmosphere. Attenuation of the solar flux is calculated using the Beer-Lambert law and takes the cosine of the Solar Zenith Angle (SZA) into account.

The actinic flux $I(\lambda, z)$ is the number of solar photons of wavelength $\lambda$ at altitude $z$ per unit of wavelength. It is calculated as:

$$
I(\lambda, z)=I_{0}(\lambda) e^{-\tau(\lambda, z)}
$$

where $I_{0}(\lambda)$ is the number of solar photons of wavelength $\lambda$ at the top of the atmosphere per unit of wavelength. The total optical depth at wavelength $\lambda$ and altitude $z$ is given by:

$$
\tau(\lambda, z)=\frac{1}{\cos (\theta)} \sum_{i} \sigma_{i}(\lambda) \int_{z}^{\infty} n_{i}\left(z^{\prime}\right) d z^{\prime}
$$

where $\sigma_{i}(\lambda)$ is the photoabsorption cross section of the species $i$ at wavelength $\lambda, n_{i}(z)$ the density of species $i$ at altitude $z$ and $\theta$ the SZA. 


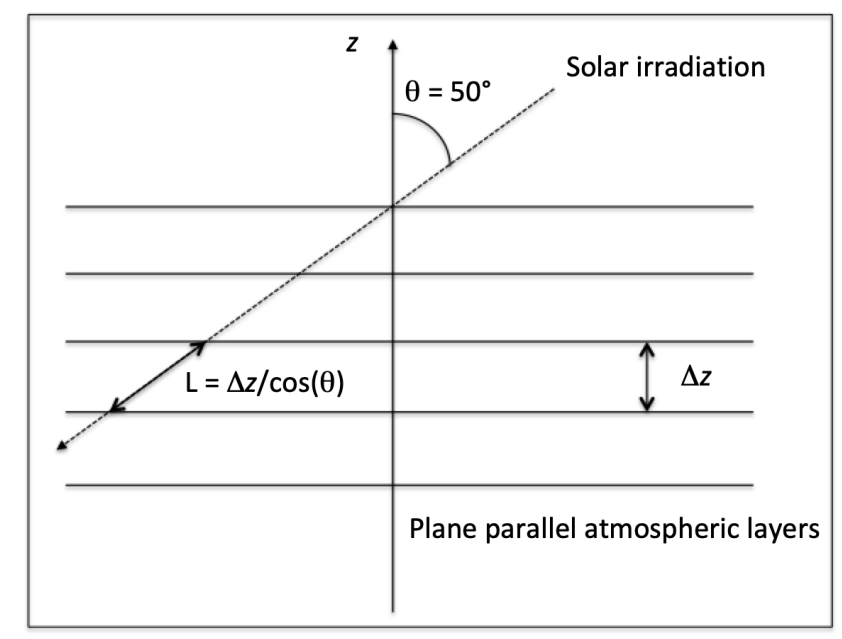

Fig. 1. Model A. The atmosphere of Titan is divided in 137 plane-parallel layers in altitude from the ground $z_{0}=0$ up to the top of the atmospheric model $z_{\text {top }}=1500$ $\mathrm{km}$. The altitude grid is non-uniform (i.e. the thickness $\Delta z$ is not constant with altitude). We use a Solar Zenith Angle $\theta=50^{\circ}$ corresponding to the mean SZA over a day at equinoxe.

The photolysis rate $J_{i}^{j}(z)$ of a species $i$ and branching path $j$ at altitude $z$ is given by:

$$
J_{i}^{j}(z)=\int q_{i}^{j}(\lambda) \sigma_{j}(\lambda) I(\lambda, z) d \lambda
$$

where $\sigma_{j}(\lambda)$ is the absorption (or ionisation) cross section and $q_{i}^{j}(\lambda)$ the branching ratio for path $j$. The total photolysis rate $J_{i}(z)$ for species $i$ is then given by:

$$
J_{i}(z)=\sum_{j=0}^{N_{j}} J_{i}^{j}(z)
$$

where $N_{j}$ is the number of paths for the photolysis process in question.

To reproduce global mean conditions, all photolysis rates are calculated for a SZA of $50^{\circ}$ (this angle is the mean SZA during the day at the equinox) and the solar flux at the top of the atmosphere is divided by 2 for day/night averaging. This approximation is also used in many other previous models but with sightly different SZA values (see for instance Hörst et al. (2008), Krasnopolsky (2009), Dobrijevic et al. (2014), Vuitton et al. (2019)). This model is called Model A in the following.

The solar irradiance at the top of the atmosphere comes from Dobrijevic et al. (2016). We consider a wavelength range from 0 to $730 \mathrm{~nm}$ with a spectral resolution of $0.001 \mathrm{~nm}$ in order to take into account the high resolution cross 
sections of many species $\left(\mathrm{N}_{2}, \mathrm{H}_{2}\right.$, CO, etc.). The model takes into account absorption, ionization, and Rayleigh scattering by $\mathrm{N}_{2}, \mathrm{H}_{2}$ and attenuation by aerosols in the lower stratosphere.

\subsection{Actinic flux in a 2D geometry}

We now present the model to compute the actinic flux in a 2D geometry. For that, we use a classical spatial discretization. The latitudinal discretization is based on a regular grid with $10^{\circ}$ separation as illustrated in Figure 2. The origin of latitudes is associated with the sub-solar point (or sub-solar latitude $0^{\circ}$, SSL0 in the following). The atmosphere is then divided in 19 sections of $10^{\circ}$ from $-5^{\circ}$ to $185^{\circ}$ sub-solar latitude (SSL). Taking both the altitudinal grid into account and latitudinal sections, the atmosphere is divided in $19 \times 138=$ 2622 cells.

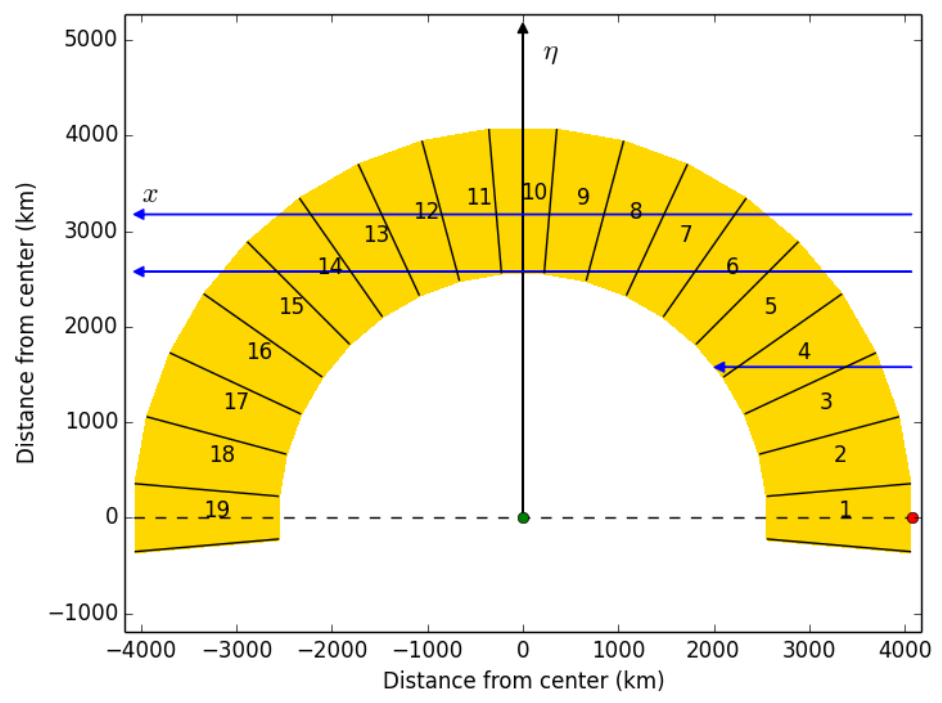

Fig. 2. Model B. The atmosphere of Titan (in yellow) is divided in 19 latitude sections from $-5^{\circ}$ to $185^{\circ}$. Each section corresponds to a latitudinal range of $10^{\circ}$. The red point corresponds to a sub-solar latitude of $0^{\circ}$ (SSL0). Section \#1 is the sub-solar section and section \#10 corresponds to SSL90. The green point corresponds to the center of Titan. The radius of Titan is $2575 \mathrm{~km}$ and the top level of altitude is 1500 $\mathrm{km}$ from the surface. The altitude grid is not depicted. The blue lines correspond to some solar rays coming from the right of the picture and going through the atmosphere.

The atmospheric vertical structure (temperature-pressure profile) and the altitudinal abundance profiles of each species are considered to be the same in all latitudinal sections.

We used a ray-tracing based method to compute the actinic flux in each lati- 
tudinal section. For a given wavelength, each solar ray goes through the atmosphere (like the ones represented by a blue line in Figure 1) and is attenuated depending on the abundance of the various absorbing species and the length of the optical path in each cell.

The actinic flux $I(\lambda, \eta, x)$ is the number of solar photons of wavelength $\lambda$ per unit of wavelength at the impact parameter $\eta$ and $x$ is the abscissa along the path of the solar ray. It is calculated as:

$$
I(\lambda, \eta, x)=I_{0}(\lambda) e^{-\tau(\lambda, \eta, x)}
$$

where $I_{0}(\lambda)$ is the solar flux at the top of the atmosphere at wavelength $\lambda$ per unit of wavelength. The total optical depth at wavelength $\lambda$ and altitude $z$ is given by:

$$
\tau(\lambda, \eta)=\sum_{i} \sigma_{i}(\lambda) \int_{0}^{\infty} n_{i}(x) d x
$$

where $\sigma_{i}(\lambda)$ is the absorption cross section of the species $i$ at wavelength $\lambda$ and $n_{i}(x)$ the density of species $i$ at the abscisse $x$.

We used a non-uniform grid for the impact parameter $\eta$ (distance from the center of Titan to the top of the atmosphere corresponding to SSL90) with a constant interval of $5 \mathrm{~km}$ from the center of Titan to a distance of the impact parameter corresponding to $2500 \mathrm{~km}$ (a little bit lower than $2575 \mathrm{~km}$ that corresponds to the radius of Titan). From $2500 \mathrm{~km}$ up to the top of the atmosphere $(4075 \mathrm{~km})$, we used a constant interval of $1 \mathrm{~km}$ to ensure that each cell at the terminator is crossed by several solar rays to compute meaningful mean photolysis rates (with a minimum of 4 rays near the surface, since $\Delta_{z}=4 \mathrm{~km}$ at the lower boundary). This gives a total of 2575 levels of the impact parameter (or 2575 solar rays from SSL0 to SSL90).

For a given wavelength and impact parameter, we compute the length of the optical path of the corresponding solar ray in each cell of the different sections it goes through. From this length and the concentration of each species (given by the 1D photochemical model), we compute the optical depth in each cell. We repeat that for all impact parameters and wavelengths. With an interval of $5 \mathrm{~km}$ or $1 \mathrm{~km}$ for the impact parameter (depending on the distance from the center of Titan to the top of the atmosphere), a given cell can be crossed by several solar rays and the number of times each cell is crossed varies from one another depending on the geometry. As a consequence, the optical depth has to be normalized. We normalized the total optical depth in each cell (the sum of optical depth) by the number of times it has been crossed by a solar ray. 
Finally, after calculating the actinic flux in each cell $(z, s)$ where $z$ is the latitude and $s$ the section number, we introduce a specific photolysis rate $J_{i}(z, s)$, which corresponds to the photolysis rate as a function of altitude for each section numbers $(\# s)$. This model is called Model B in the following.

\subsection{Coupling of the UV transfer models and the photochemical model}

The first step of our calculations (step \#1 in Figure 3) is to compute the photolysis rates for Model A and the mole fraction profiles at the steady state with the 1D photochemical model. As a starting point of the Model A, we consider that the atmosphere is only composed of $\mathrm{N}_{2}, \mathrm{CH}_{4}, \mathrm{Ar}$ and $\mathrm{H}_{2}$ (the initial atmosphere). This allows computing the first photolysis rates for all photolysis (photodissociation and photoionisation) processes considered. Then, we compute the relative abundance of each species using the 1D photochemical model until we reach a steady state. We then iterate this procedure considering the whole set of photolysis processes (see Loison et al. (2019) for information on the chemical scheme) until we reach a convergence in the relative abundance profiles (in practice, two iterations are sufficient; uncertainties on reaction rates render useless obtaining a convergence criteria lower than few percent).

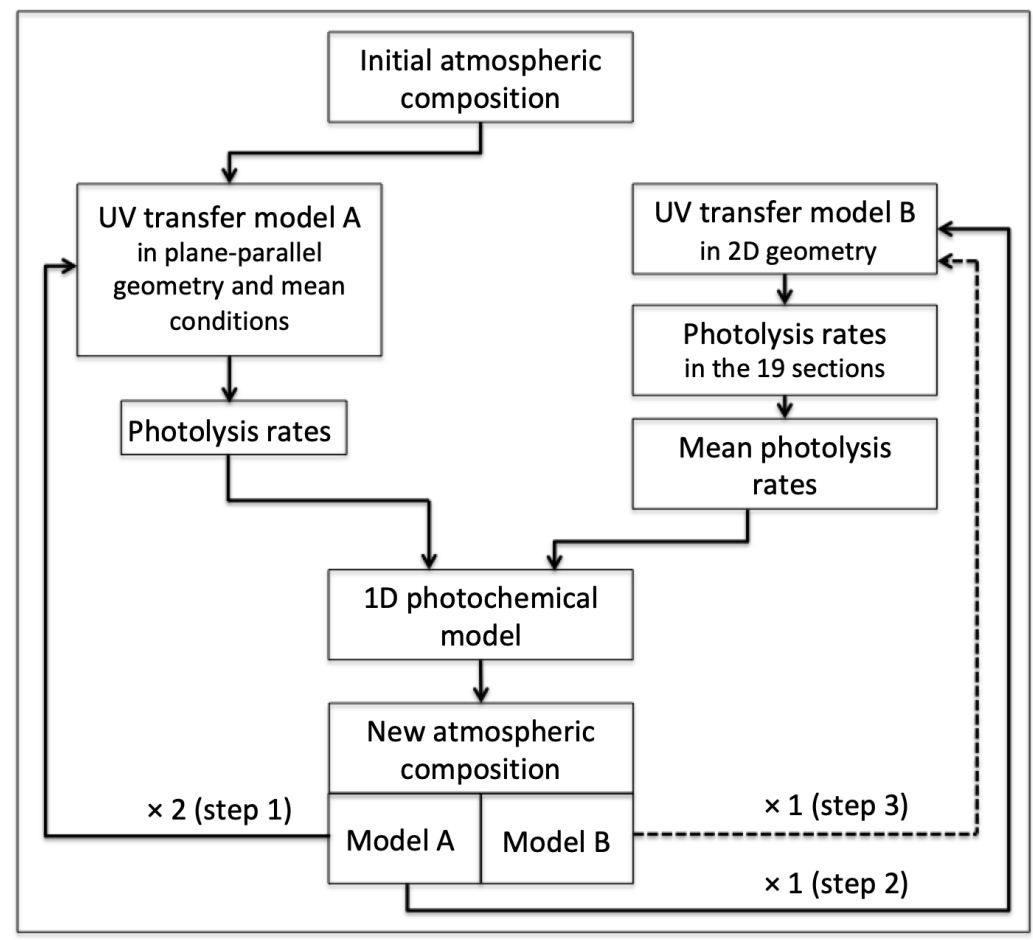

Fig. 3. Schematic diagram showing the two UV transfer models and their use with our 1D photochemical model.

In a 2D geometry for calculating the actinic flux, the mean conditions are obtained by using the photolysis rates calculated with the Model B and the 
atmospheric composition obtained with the Model A (end of step \#1). This model computes the photolysis rates for each section. Then, we average them over all the sections to obtain mean photolysis rates, which correspond to an average over SZA from $0^{\circ}$ to $90^{\circ}$ and day/night average. This procedure corresponds to step \#2 in Figure 3.

In the following section, we first present the photolysis rate results of these two approaches and then we compare the mole fraction profiles obtained with the same 1D photochemical model. A comment on step \#3 is given in the conclusion.

\section{Results: photolysis rates}

\subsection{Photolysis rates}

\subsubsection{Model A}

At the steady state, the depth of penetration of solar photons in the Model A is shown in Figure 4. This level corresponds to the altitude where the total optical depth is equal to 1 at a given wavelength (which corresponds also to the maximum of the photolysis rate). Molecular nitrogen $\left(\mathrm{N}_{2}\right)$ is the main absorber in the EUV up to $80 \mathrm{~nm}$. High resolution cross section of $\mathrm{N}_{2}$ is only known between approximately $80 \mathrm{~nm}$ and $100 \mathrm{~nm}$. From 80 to $140 \mathrm{~nm}$, methane $\left(\mathrm{CH}_{4}\right)$ absorbs with a contribution of molecular hydrogen $\left(\mathrm{H}_{2}\right)$ between 100 and $110 \mathrm{~nm}$. Around $150 \mathrm{~nm}$, both carbon monoxyde (CO) and acetylene $\left(\mathrm{C}_{2} \mathrm{H}_{2}\right)$ contribute to the absorption. Several species absorb at greater wavelengths with the major contribution of aerosols beyond $200 \mathrm{~nm}$.

\subsubsection{Model B}

Figure 5 shows the optical depth as a function of latitude and altitude at two wavelengths (85 $\mathrm{nm}$ and $121.6 \mathrm{~nm}$ ) where $\mathrm{N}_{2}$ and $\mathrm{CH}_{4}$ absorb efficiently. This figure shows also the night side where photolysis is null. We see the increase of the altitude level corresponding to the maximum of the photolysis rate (where the optical depth is equal to 1) from the SSL0 to the SSL90 and beyond. The depth of penetration of UV is lower at $85 \mathrm{~nm}$ than at $121.6 \mathrm{~nm}$. The most important difference between the 2D and 1D plane parallel models is that a substantial part of the atmosphere beyond the terminator is photochemically active, when it is by definition inactive in 1D models. This geometric difference should enhance the mean photolysis rate over the entire atmosphere. 


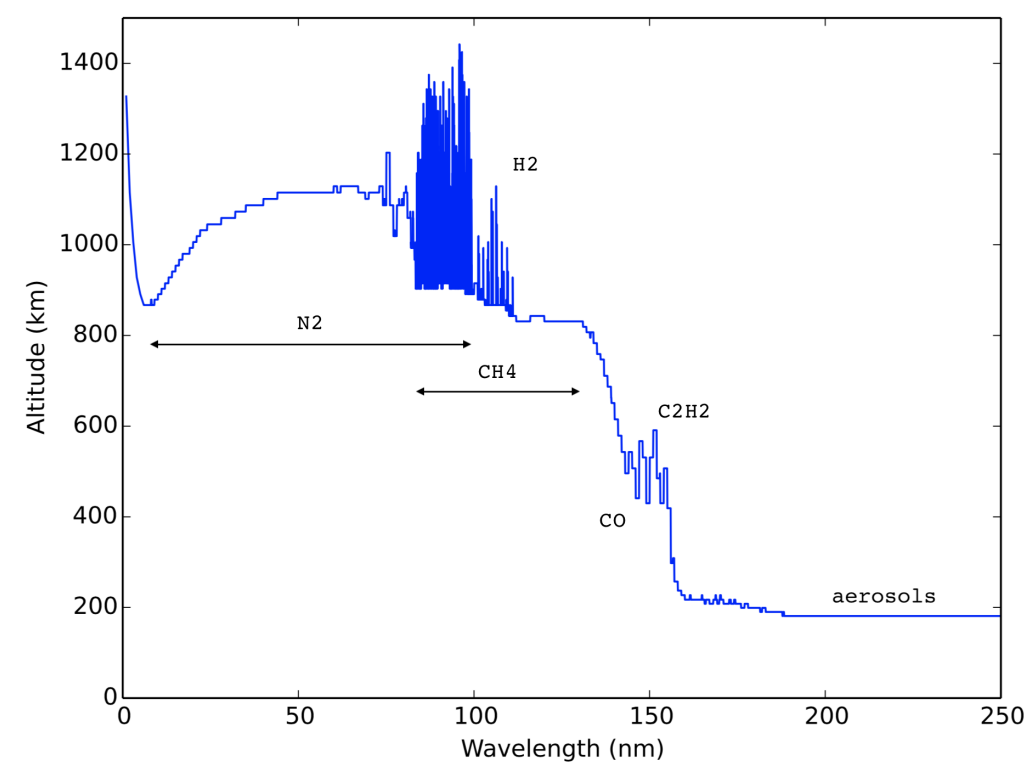

Fig. 4. Depth of penetration of solar photons in the atmosphere of Titan as a function of wavelength. The main contributors to the absorption are depicted.

Figure 6 shows an example of photolysis rates as a function of altitude and section for $\mathrm{H}_{2}$. Although the first 3 sections do not exhibit strong differences in the photodissociation rates of $\mathrm{H}_{2}$ (for a given altitude), the rate profiles change more and more strongly in the following sections. The $\mathrm{H}_{2}$ photodissociation rate decreases more rapidly and occurs at higher altitude as a function of section number toward terminator (SSL90) and beyond. The photolysis of $\mathrm{H}_{2}$ is no more efficient after section \#12 (SSL115).

Let us write $J_{i}(z, s)$ the photolysis rate of species $i$ at altitude $z$ and section number $s$. The global mean photolysis rate over the entire atmosphere $\bar{J}_{i}(z)$ is simply given by:

$$
\bar{J}_{i}(z)=\frac{\sum_{s=1}^{N_{s}} J_{i}(z, s)}{N_{s}}
$$

where $N_{s}=19$ is the number of sections considered in Model B. Figure 6 shows the mean rate $\bar{J}_{i}(z)$ for $\mathrm{H}_{2}$ obtained from equation 7 . The effect of day/night mean is clearly visible.

\subsubsection{Validation}

The photolysis rates are calculated in two different ways in models A and B. In order to validate the two radiative transfer models and to highlight the geometric effect on the calculation of photolysis rates, we first compared 

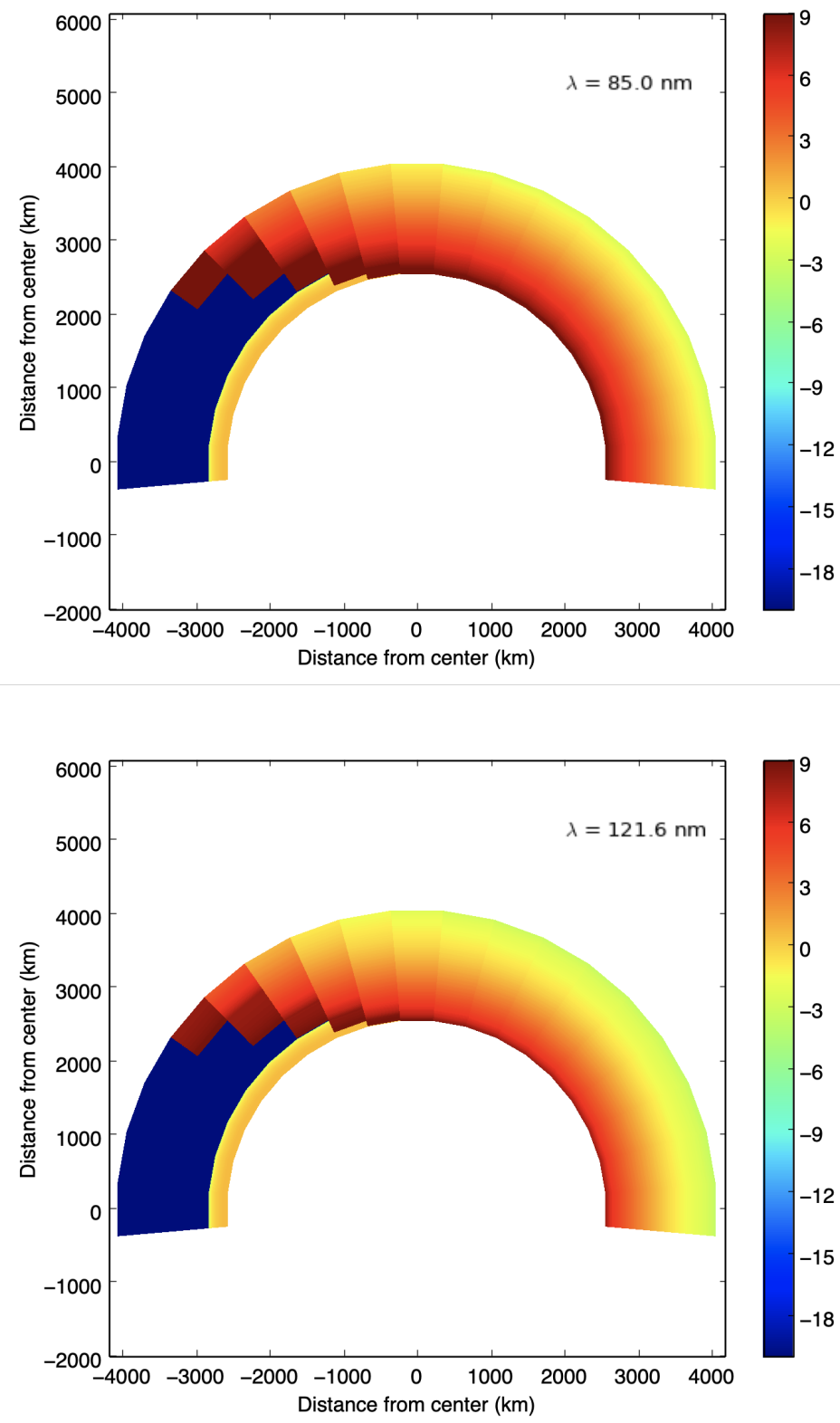

Fig. 5. Logarithm of the total optical depth as a function of altitude and section at two wavelengths $(85 \mathrm{~nm}$ and $121.6 \mathrm{~nm}$ ). The maximum of the photolysis rate profile corresponds to the altitude where the total optical depth is equal to 1 (altitude level corresponding to the orange level). The contribution of aerosols extinction to the total optical depth is visible on the night side (but it plays no role on this side).

the rates obtained with Model $\mathrm{A}\left(J_{A}\right)$ considering a $\mathrm{SZA}=0^{\circ}$ and Model $\mathrm{B}$ $\left(J_{B}\right)$ after the calculation of the SSL0 mean rates obtained with sections \#1 and \#19. In Model A, the solar flux at the top of the atmosphere is divided by 2 for day/night averaging. In Model B, since the actinic flux is null in section \#19, the averaging of sections \#1 and \#19 corresponds to a division of the photolysis rates in section \#1 by a factor of 2 . Results are presented 


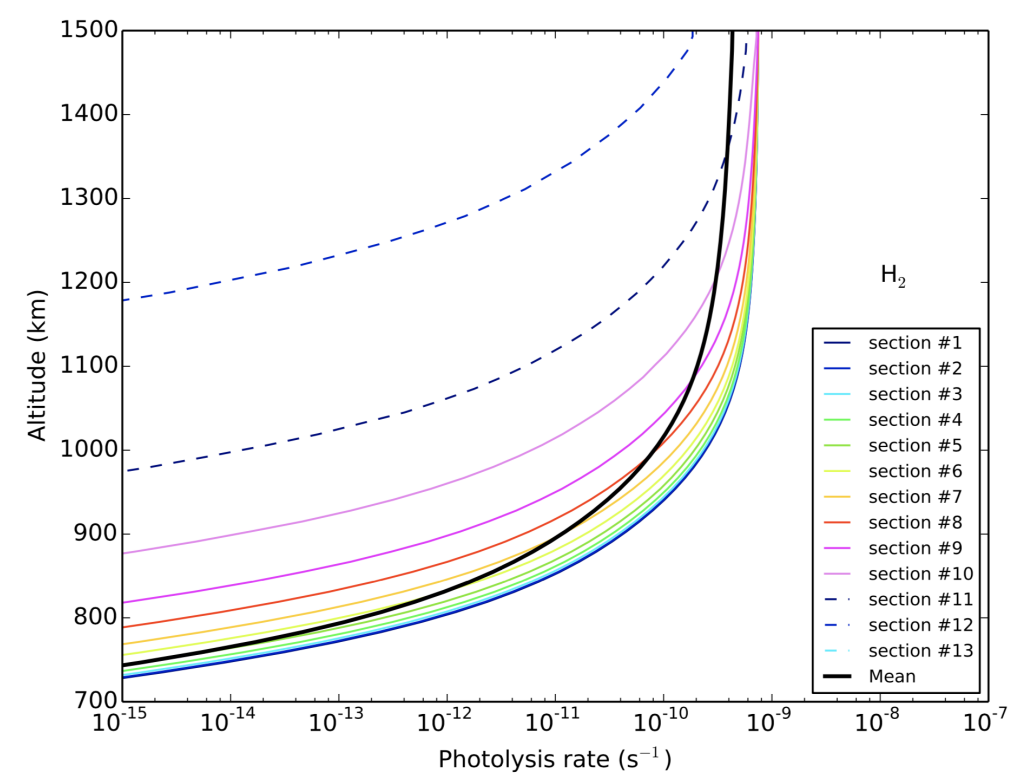

Fig. 6. Photolysis rate of $\mathrm{H}_{2}$ as a function of altitude and section number. The rate of section \#13 and following sections are too low and does not appear in the figure. The mean photolysis rate computed from all the sections is shown in black for comparison.

in Figure 7. For a species like $\mathrm{CH}_{4}$ with a low resolution cross section, the agreement between the two models is perfect. Note that, even for section \#1 in Model B, there is a geometry effect that is not taken into account in planeparallel models (Model A). For $\mathrm{N}_{2}$ (not shown), the ratio $J_{B} / J_{A}$ does not exceed 1.1 above $600 \mathrm{~km}$ of altitude (only a fraction of the cross section is at high resolution). On the other hand, for species with high resolution cross sections, there are noticeable differences as a function of altitude. For CO (not shown), the ratio $\left(J_{B} / J_{A}\right)$ is about 0.7 between 800 and $1000 \mathrm{~km}$. This is due to the fact that at some specific wavelength photons go deeper (or are stopped higher) in the atmosphere and the geometric effect in the 2D model becomes more significant. In Model B, photons tend to be slightly absorbed at higher altitude than in Model A. This difference increases when comparing Model A with the other sections in Model B (sections \#2 and \#18 for instance). For $\mathrm{H}_{2}$, the effect is the opposite, $J_{A}$ being about $40 \%$ greater than $J_{B}$ at $900 \mathrm{~km}$ of altitude (see Figure 7).

Due to the geometry of the 2D model, the best agreement between the 1D model at mean conditions (Model A) and the 2D model with day/night averaged rates (Model B) is obtained when considering section \#7 (between SSL55 and SSL65) and section \#13 (which is the symmetric of section \#7 with respect to the terminator). This result is in agreement with the use of a SZA equal to $60^{\circ}$ in $1 \mathrm{D}$ photochemical models in global mean conditions. 

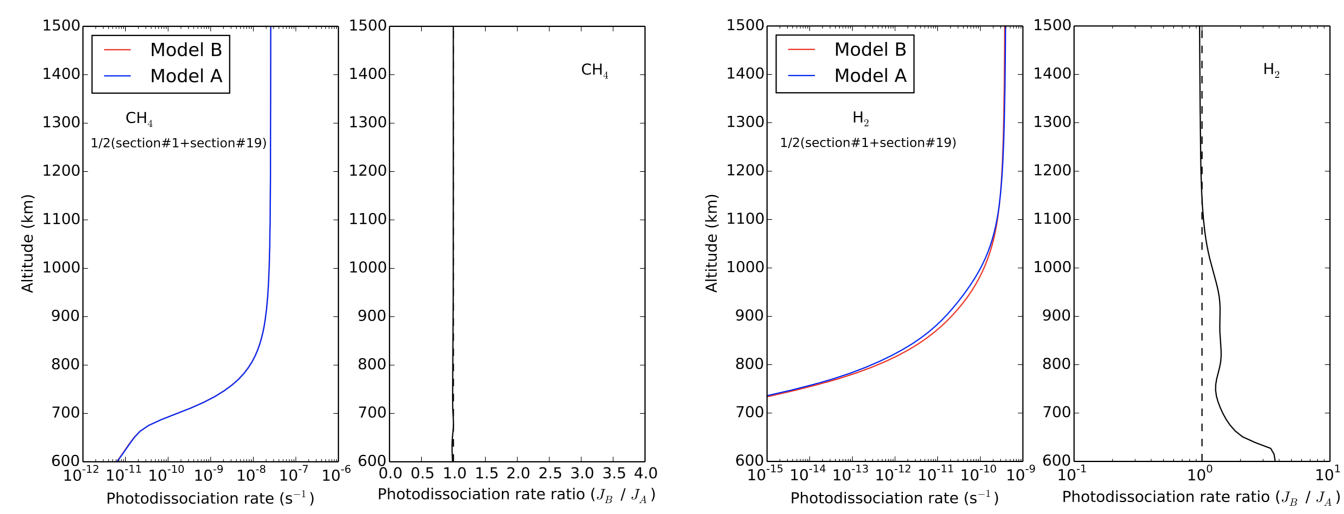

Fig. 7. Left sub-panels: Photolysis rate of $\mathrm{CH}_{4}$ and $\mathrm{H}_{2}$ for a SZA $=0^{\circ}\left(J_{A}\right.$, Model A) and for the mean of sections \#1 and \#19 ( $J_{B}$, Model B). Right sub-panels: Ratios of the photolysis rates $\left(J_{B} / J_{A}\right)$.

\subsubsection{Comparison between Model $A$ and Model B}

We present in Figure 8 the global mean photolysis rates of the two major compounds in the photochemistry of Titan's atmosphere. The maximum of methane photolysis occurs around an altitude of $800 \mathrm{~km}$ (see Figure 4). At this altitude, the ratio $J_{B} / J_{A}$ is around 1.3 and increases up to 2.7 at $735 \mathrm{~km}$. For $\mathrm{N}_{2}$, the ratio $J_{B} / J_{A}$ is between 1.1 and 1.5 in the altitudinal range [1100,900] $\mathrm{km}$ where $\mathrm{N}_{2}$ is efficiently photolyzed. As a consequence, the two precursor mechanisms of chemical complexity are increased in Model B compared to Model A. We see in the next section that this has a noticeable effect on the production of organic compounds in the atmosphere.
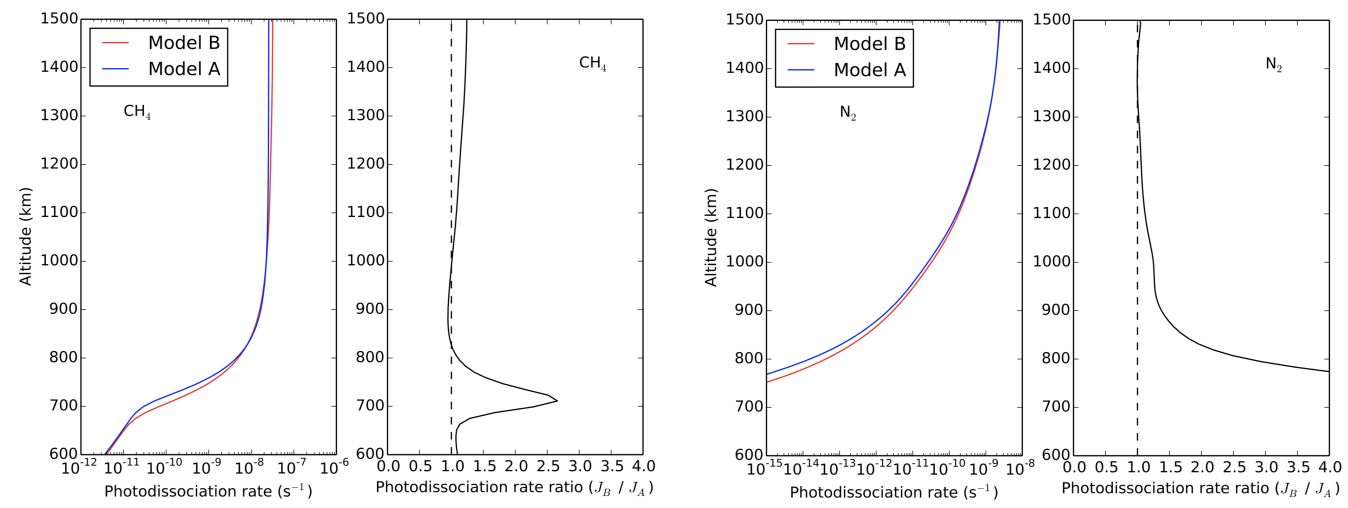

Fig. 8. Global mean photolysis rate of $\mathrm{CH}_{4}$ and $\mathrm{N}_{2}$ for Model $\mathrm{A}\left(J_{A}\right)$ and for Model B $\left(J_{B}\right)$. Right sub-panels: Ratios of the photolysis rates $\left(J_{B} / J_{A}\right)$.

We can also compare the partial photolysis rates for the various path of photodissociation of a given species. For instance, in Figure 9, we present the total photolysis rates of $\mathrm{C}_{2} \mathrm{H}_{6}$ for the two models. We see that these rates are quite identical throughout the atmosphere. However, the ratios $J_{B}(i) / J_{A}(i)$ for the different pathways of photodissociation are very different above 800 
$\mathrm{km}$. Paths 1 and 3 (to a lesser extent) are the major contributors to the total photodissociation rate. Their ratios are counterbalanced by the ones of paths 2, 4 and 5. This example shows that partial rates of paths 1 and 3 are favored in Model B in contrast to paths 2, 4 and 5.

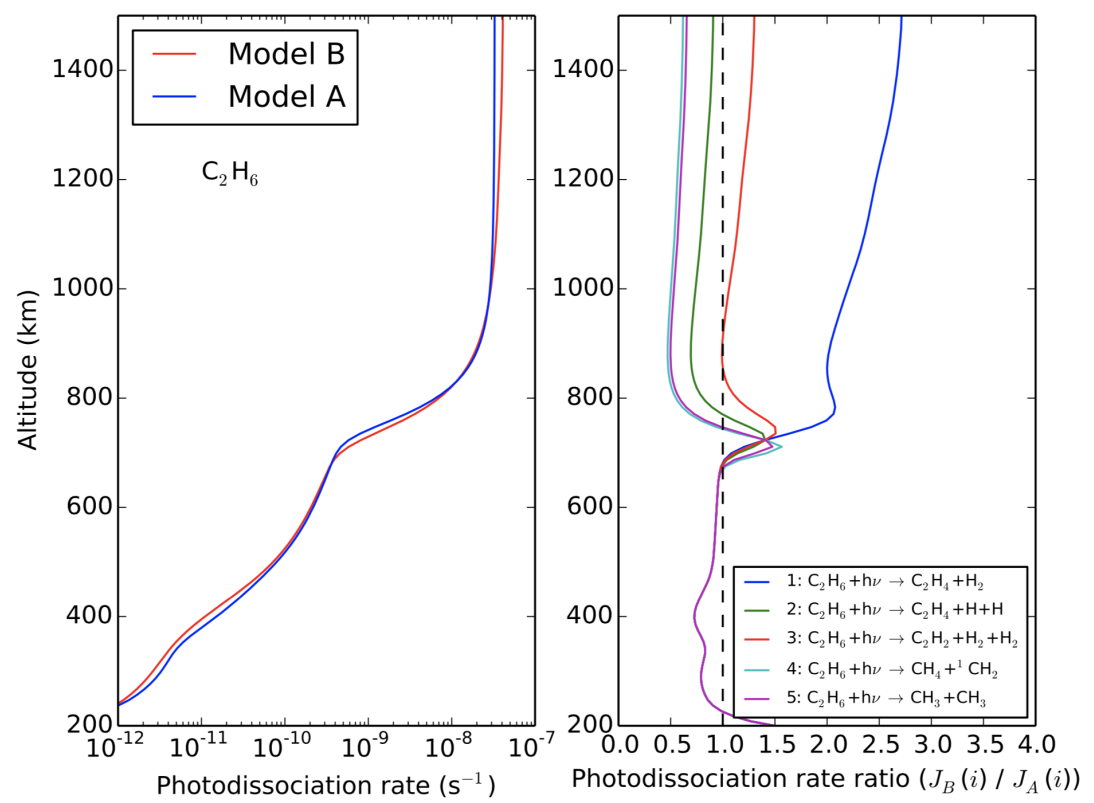

Fig. 9. Left: Photolysis rates of $\mathrm{C}_{2} \mathrm{H}_{6}$ for Models A and B. Right: Photolysis rate ratios for the five photodissociation pathways.

In order to give an overview of the impact of the $2 \mathrm{D}$ geometry on the calculation of photolysis rates, we compare the two models in Tables 1 and 2 for each photolysis process included in our model. In these tables, we selected the following quantities:

- The number of compounds that are photodissociated (or photoionised) $P_{i}(z)$ at altitude $z\left(\right.$ in $\left.\mathrm{cm}^{-3} \mathrm{~s}^{-1}\right)$ is given by:

$$
P_{i}(z)=n_{i}(z) \times J_{i}(z)
$$

where $n_{i}(z)$ is the density of compound $i\left(\right.$ in $\left.\mathrm{cm}^{-3}\right)$ and $J_{i}(z)$ its photodissociation (or photoionisation) rate $\left(\right.$ in $^{-1}$ ) at altitude $z$. This quantity reaches a maximum $P_{i}\left(z_{m}\right)$ at altitude $z_{m}$. For instance, the maximum of methane photodissociation occurs around an altitude of $830 \mathrm{~km}$ with 42.42 molecules dissociated per $\mathrm{cm}^{3}$ and per second. In the tables, the subscripts $\mathrm{A}$ and $\mathrm{B}$ correspond to Model A and Model B respectively.

- The ratio $P_{B}\left(z_{m, B}\right) / P_{A}\left(z_{m, A}\right)$ is given for the altitude corresponding the maximum of $P_{i}(z), z_{m, A}$ in Model A and $z_{m, B}$ in Model B. We see in the tables that $z_{m, B}$ can be at the same altitude than in Model $\mathrm{A}$ but in general, it is at lower altitude, and sometimes at higher altitude (like for $\mathrm{C}_{2} \mathrm{H}_{4}$ ). The precision on $z_{m, A}$ and $z_{m, B}$ is limited by the discretization of our alti- 
tude grid. For instance, around $900 \mathrm{~km}$ of altitude the interval between two consecutive altitude levels is $12 \mathrm{~km}$ and $10 \mathrm{~km}$ around $220 \mathrm{~km}$ of altitude.

For some processes, the peak of dissociation is quite pronounced and the ratio $P_{B}\left(z_{m, B}\right) / P_{A}\left(z_{m, A}\right)$ is rather representative of the difference between the two models. For others processes, the dissociation occurs in a larger part of the atmosphere and it is therefore instructive to define the column-integrated production rate, such as:

$$
\widetilde{P}_{i}=\int_{0}^{z_{t o p}} P_{i}(z) d z
$$

The integration is done from the surface to the top of our atmospheric model. For instance, the ratio $\widetilde{P}_{B} / \widetilde{P}_{A}$ is 1.12 for $\mathrm{C}_{2} \mathrm{H}_{2}$ and only 1.01 for $P_{B}\left(z_{m, B}\right) /$ $P_{A}\left(z_{m, A}\right)$.

The general trend is the following. With very few exceptions (see $\mathrm{C}_{2} \mathrm{H}_{6}$, $\mathrm{CH}_{3} \mathrm{C}_{3} \mathrm{~N}$ and $\mathrm{CO}$ ), the photodissociation rates are enhanced in Model $\mathrm{B}$ compared to Model A. The enhancement is between $10 \%$ and $30 \%$. There is no systematic correlation between this quantity and the altitude of the peak of photodissociation. The most affected rate is the one of $\mathrm{HCO}$ for which the peak of photodissociation is very low in the stratosphere (around 120-140 km) with an enhancement factor of 2.4 at the peak. Also, there is no clear correlation between the value of the ratio $\widetilde{P}_{B} / \widetilde{P}_{A}$ and the value of $\widetilde{P}_{A}$.

Results for photoionisation rates are presented in Table 2. They are significantly different from the one obtained for photodissocation rates. For some species, the photoionisation rates are lower in Model B (this is the case for $\mathrm{H}$ and $\mathrm{C}_{4} \mathrm{H}_{2}$ ), quite identical in both models, or even enhanced in Model $\mathrm{B}$ (for $\mathrm{C}_{6} \mathrm{H}_{6}^{*}, \mathrm{CO}_{2}$ and $\mathrm{CO}$ ). In general, the peak of photolysis occurs at lower altitudes in Model B.

\section{Results: abundance profiles}

We have seen in the previous section that the geometric effects alter both the magnitude of the rates, the relative importance of the branching paths and the location of the peak of photolysis. We now present how these differences in photolysis rate profiles affect the abundances of the various species calculated from the two models. It is not possible to show the abundance profiles of the 182 species present in our model so we focus our presentation on some particular species, especially some of those that have been detected. 
Table 1

Report of photodissocation rate results for Models A and B. $P_{A}\left(z_{m, A}\right)$ is the maximum of the number of compounds that are photodissociated in $\mathrm{cm}^{-3} \mathrm{~s}^{-1}$ at altitude $z_{m, A}$ for Model A. $\widetilde{P}_{A}$ is the column-integrated production rate for Model A in $\mathrm{cm}^{-2} \mathrm{~S}^{-1}$

\begin{tabular}{|c|c|c|c|c|c|c|}
\hline Compound & $\begin{array}{c}P_{A}\left(z_{m}, A\right) \\
\left(\mathrm{cm}^{-3} \mathrm{~s}^{-1}\right)\end{array}$ & $\begin{array}{c}z_{m, A} \\
(\mathrm{~km})\end{array}$ & $\begin{array}{r}z_{m, B} \\
(\mathrm{~km})\end{array}$ & $P_{B}\left(z_{m, B}\right) / P_{A}\left(z_{m, A}\right)$ & $\begin{array}{c}\widetilde{P}_{A} \\
\left(\mathrm{~cm}^{-2} \mathrm{~s}^{-1}\right)\end{array}$ & $\widetilde{P}_{B} / \widetilde{P}_{A}$ \\
\hline $\mathrm{H}_{2}$ & $4.12 \times 10^{-3}$ & 1019 & 980 & 1.36 & $1.15 \times 10^{5}$ & 1.30 \\
\hline $\mathrm{CH}_{3}$ & $1.11 \times 10^{1}$ & 807 & 807 & 1.13 & $3.75 \times 10^{8}$ & 1.10 \\
\hline $\mathrm{CH}_{4}$ & $4.24 \times 10^{1}$ & 831 & 819 & 1.00 & $8.25 \times 10^{8}$ & 1.08 \\
\hline $\mathrm{C}_{2} \mathrm{H}_{2}$ & $2.34 \times 10^{2}$ & 227 & 217 & 1.01 & $3.39 \times 10^{9}$ & 1.12 \\
\hline $\mathrm{C}_{2} \mathrm{H}_{3}$ & $4.36 \times 10^{-1}$ & 227 & 227 & 1.03 & $5.28 \times 10^{6}$ & 1.12 \\
\hline $\mathrm{C}_{2} \mathrm{H}_{4}$ & $1.78 \times 10^{1}$ & 227 & 651 & 0.95 & $9.45 \times 10^{8}$ & 1.00 \\
\hline $\mathrm{C}_{2} \mathrm{H}_{6}$ & $1.68 \times 10^{-1}$ & 257 & 247 & 0.83 & $6.87 \times 10^{6}$ & 0.94 \\
\hline $\mathrm{C}_{3}$ & $8.57 \times 10^{-4}$ & 1059 & 1073 & 1.17 & $2.04 \times 10^{4}$ & 1.16 \\
\hline $\mathrm{cC}_{3} \mathrm{H}_{2}$ & $9.70 \times 10^{-1}$ & 891 & 891 & 1.32 & $3.37 \times 10^{7}$ & 1.33 \\
\hline $\mathrm{C}_{3} \mathrm{H}_{3}$ & $1.16 \times 10^{0}$ & 208 & 199 & 1.29 & $3.29 \times 10^{7}$ & 1.36 \\
\hline $\mathrm{CH}_{3} \mathrm{C}_{2} \mathrm{H}$ & $5.10 \times 10^{0}$ & 208 & 199 & 1.08 & $6.17 \times 10^{7}$ & 1.18 \\
\hline $\mathrm{CH}_{2} \mathrm{CCH}_{2}$ & $3.06 \times 10^{0}$ & 217 & 208 & 1.05 & $5.21 \times 10^{7}$ & 1.17 \\
\hline $\mathrm{C}_{3} \mathrm{H}_{6}$ & $1.45 \times 10^{1}$ & 217 & 199 & 1.06 & $1.59 \times 10^{8}$ & 1.18 \\
\hline $\mathrm{C}_{3} \mathrm{H}_{8}$ & $8.98 \times 10^{-1}$ & 237 & 237 & 0.94 & $1.33 \times 10^{7}$ & 0.99 \\
\hline $\mathrm{C}_{4} \mathrm{H}_{2}$ & $1.86 \times 10^{1}$ & 217 & 208 & 1.15 & $2.89 \times 10^{8}$ & 1.27 \\
\hline $\mathrm{C}_{4} \mathrm{H}_{4}$ & $4.13 \times 10^{0}$ & 227 & 227 & 1.07 & $7.29 \times 10^{7}$ & 1.19 \\
\hline $\mathrm{C}_{4} \mathrm{H}_{6}$ & $1.15 \times 10^{1}$ & 190 & 181 & 1.32 & $1.23 \times 10^{8}$ & 1.40 \\
\hline $\mathrm{C}_{4} \mathrm{H}_{8}$ & $1.03 \times 10^{1}$ & 208 & 190 & 1.15 & $1.08 \times 10^{8}$ & 1.25 \\
\hline $\mathrm{C}_{4} \mathrm{H}_{10}$ & $1.46 \times 10^{0}$ & 237 & 227 & 0.97 & $1.97 \times 10^{7}$ & 1.04 \\
\hline $\mathrm{C}_{6} \mathrm{H}_{6}$ & $2.69 \times 10^{1}$ & 267 & 277 & 1.04 & $8.91 \times 10^{8}$ & 1.21 \\
\hline $\mathrm{C}_{6} \mathrm{H}_{6}^{*}$ & $2.68 \times 10^{-1}$ & 891 & 891 & 1.32 & $8.24 \times 10^{6}$ & 1.32 \\
\hline $\mathrm{C}_{6} \mathrm{H}_{5} \mathrm{CH}_{3}$ & $2.78 \times 10^{1}$ & 247 & 247 & 1.05 & $4.48 \times 10^{8}$ & 1.16 \\
\hline $\mathrm{C}_{6} \mathrm{H}_{5} \mathrm{C}_{2} \mathrm{H}$ & $1.51 \times 10^{-2}$ & 639 & 651 & 1.26 & $7.79 \times 10^{5}$ & 1.27 \\
\hline $\mathrm{C}_{6} \mathrm{H}_{5} \mathrm{C}_{2} \mathrm{H}_{5}$ & $6.27 \times 10^{0}$ & 217 & 208 & 1.12 & $8.07 \times 10^{7}$ & 1.25 \\
\hline $\mathrm{C}_{6} \mathrm{H}_{5} \mathrm{NH}_{2}$ & $2.60 \times 10^{-2}$ & 419 & 419 & 1.19 & $1.07 \times 10^{6}$ & 1.25 \\
\hline $\mathrm{C}_{6} \mathrm{H}_{5} \mathrm{NHCH}_{3}$ & $2.37 \times 10^{-3}$ & 993 & 993 & 1.32 & $5.93 \times 10^{4}$ & 1.29 \\
\hline $\mathrm{C}_{6} \mathrm{H}_{5} \mathrm{CN}$ & $4.99 \times 10^{-4}$ & 855 & 855 & 1.35 & $1.80 \times 10^{4}$ & 1.29 \\
\hline AROM & $3.71 \times 10^{0}$ & 199 & 181 & 1.33 & $4.46 \times 10^{7}$ & 1.4 \\
\hline $\mathrm{NH}_{2}$ & $1.17 \times 10^{-3}$ & 639 & 639 & 1.15 & $4.55 \times 10^{4}$ & 1.17 \\
\hline $\mathrm{NH}_{3}$ & $9.86 \times 10^{-3}$ & 879 & 879 & 1.30 & $3.94 \times 10^{5}$ & 1.29 \\
\hline $\mathrm{N}_{2}$ & $6.77 \times 10^{-1}$ & 1087 & 1073 & 1.14 & $2.42 \times 10^{7}$ & 1.14 \\
\hline $\mathrm{HCN}$ & $2.22 \times 10^{0}$ & 227 & 217 & 1.06 & $3.33 \times 10^{7}$ & 1.16 \\
\hline $\mathrm{HNC}$ & $1.04 \times 10^{-2}$ & 967 & 967 & 0.99 & $2.41 \times 10^{5}$ & 1.02 \\
\hline $\mathrm{CH}_{2} \mathrm{NH}$ & $1.28 \times 10^{-1}$ & 831 & 831 & 1.34 & $5.28 \times 10^{6}$ & 1.33 \\
\hline $\mathrm{CH}_{3} \mathrm{NH}_{2}$ & $8.38 \times 10^{-2}$ & 485 & 485 & 1.20 & $1.98 \times 10^{6}$ & 1.20 \\
\hline $\mathrm{CH}_{3} \mathrm{CN}$ & $1.15 \times 10^{-1}$ & 227 & 217 & 1.05 & $1.75 \times 10^{6}$ & 1.14 \\
\hline $\mathrm{HC}_{3} \mathrm{~N}$ & $5.57 \times 10^{-2}$ & 639 & 639 & 1.20 & $2.63 \times 10^{6}$ & 1.20 \\
\hline $\mathrm{HC}_{5} \mathrm{~N}$ & $1.48 \times 10^{-2}$ & 867 & 867 & 1.18 & $3.68 \times 10^{5}$ & 1.17 \\
\hline $\mathrm{C}_{2} \mathrm{H}_{3} \mathrm{CN}$ & $2.72 \times 10^{-1}$ & 519 & 519 & 1.22 & $1.41 \times 10^{7}$ & 1.24 \\
\hline $\mathrm{C}_{2} \mathrm{H}_{5} \mathrm{CN}$ & $6.18 \times 10^{-2}$ & 227 & 217 & 1.05 & $1.03 \times 10^{6}$ & 1.13 \\
\hline $\mathrm{C}_{3} \mathrm{H}_{7} \mathrm{CN}$ & $1.68 \times 10^{-3}$ & 227 & 217 & 1.00 & $2.25 \times 10^{4}$ & 1.10 \\
\hline $\mathrm{CH}_{3} \mathrm{C}_{3} \mathrm{~N}$ & $6.65 \times 10^{-2}$ & 843 & 855 & 0.92 & $1.67 \times 10^{6}$ & 0.92 \\
\hline $\mathrm{C}_{2} \mathrm{~N}_{2}$ & $7.67 \times 10^{-3}$ & 247 & 247 & 0.95 & $2.47 \times 10^{5}$ & 1.06 \\
\hline $\mathrm{C}_{4} \mathrm{~N}_{2}$ & $1.63 \times 10^{-3}$ & 795 & 795 & 1.12 & $6.51 \times 10^{4}$ & 1.12 \\
\hline $\mathrm{H}_{2} \mathrm{O}$ & $4.05 \times 10^{-2}$ & 247 & 247 & 0.98 & $1.02 \times 10^{6}$ & 1.10 \\
\hline $\mathrm{CO}$ & $2.80 \times 10^{-3}$ & 891 & 879 & 0.79 & $6.29 \times 10^{4}$ & 0.85 \\
\hline $\mathrm{HCO}$ & $3.47 \times 10^{0}$ & 147 & 123 & 2.44 & $3.83 \times 10^{7}$ & 2.25 \\
\hline $\mathrm{H}_{2} \mathrm{CO}$ & $1.12 \times 10^{-2}$ & 855 & 855 & 1.35 & $5.32 \times 10^{5}$ & 1.34 \\
\hline $\mathrm{CO}_{2}$ & $4.02 \times 10^{-3}$ & 227 & 217 & 1.08 & $5.30 \times 10^{4}$ & 1.18 \\
\hline
\end{tabular}


Table 2

Report of photoionisation rate results for Models A and B. $P_{A}\left(z_{m, A}\right)$ is the maximum of the number of compounds that are photoionised in $\mathrm{cm}^{-3} \mathrm{~s}^{-1}$ at altitude $z_{m, A}$ for Model A. $\widetilde{P}_{A}$ is the column-integrated production rate for Model A in $\mathrm{cm}^{-2} \mathrm{~s}^{-1}$.

\begin{tabular}{|c|c|c|c|c|c|c|}
\hline Compound & $\begin{array}{c}P_{A}\left(z_{m, A}\right) \\
\left(\mathrm{cm}^{-3} \mathrm{~s}^{-1}\right)\end{array}$ & $\begin{array}{c}z_{m, A} \\
(\mathrm{~km})\end{array}$ & $\begin{array}{c}z_{m, B} \\
(\mathrm{~km})\end{array}$ & $\begin{array}{c}P_{B}\left(z_{m, B}\right) / P_{A}\left(z_{m, A}\right) \\
\widetilde{P}_{A} \\
\left(\mathrm{~cm}^{-2} \mathrm{~s}^{-1}\right)\end{array}$ & $\widetilde{P}_{B} / \widetilde{P}_{A}$ \\
\hline $\mathrm{H}$ & $9.60 \times 10^{-4}$ & 954 & 928 & 0.70 & $2.09 \times 10^{4}$ & 0.79 \\
$\mathrm{H}_{2}$ & $1.87 \times 10^{-3}$ & 1101 & 1087 & 1.06 & $4.66 \times 10^{4}$ & 1.12 \\
$\mathrm{~N}_{2}$ & $3.40 \times 10^{0}$ & 1073 & 1059 & 1.02 & $7.98 \times 10^{7}$ & 1.07 \\
$\mathrm{CH}_{3}$ & $2.20 \times 10^{-2}$ & 879 & 879 & 0.89 & $4.90 \times 10^{5}$ & 0.94 \\
$\mathrm{CH}_{4}$ & $7.75 \times 10^{-1}$ & 954 & 915 & 0.83 & $2.27 \times 10^{7}$ & 0.95 \\
$\mathrm{cC}_{3} \mathrm{H}_{2}$ & $7.14 \times 10^{-4}$ & 980 & 993 & 1.00 & $1.95 \times 10^{4}$ & 1.02 \\
$\mathrm{CH}_{3} \mathrm{C}_{2} \mathrm{H}$ & $1.84 \times 10^{-3}$ & 928 & 915 & 0.91 & $4.42 \times 10^{4}$ & 0.95 \\
$\mathrm{CH}_{2} \mathrm{CCH}_{2}$ & $1.12 \times 10^{-3}$ & 954 & 941 & 0.89 & $2.70 \times 10^{4}$ & 0.94 \\
$\mathrm{C}_{4} \mathrm{H}_{2}$ & $2.29 \times 10^{-2}$ & 879 & 879 & 0.5 & $4.37 \times 10^{5}$ & 0.53 \\
$\mathrm{C}_{6} \mathrm{H}_{6}$ & $1.84 \times 10^{-3}$ & 855 & 843 & 0.98 & $3.20 \times 10^{4}$ & 1.04 \\
$\mathrm{C}_{6} \mathrm{H}_{6}^{*}$ & $1.61 \times 10^{-2}$ & 891 & 891 & 1.34 & $4.95 \times 10^{5}$ & 1.33 \\
$\mathrm{C}_{6} \mathrm{H}_{5} \mathrm{CH}_{3}$ & $1.58 \times 10^{-3}$ & 879 & 879 & 0.95 & $2.81 \times 10^{4}$ & 1.00 \\
$\mathrm{C}_{6} \mathrm{H}_{5} \mathrm{C}_{2} \mathrm{H}_{5}$ & $1.99 \times 10^{-4}$ & 903 & 903 & 0.95 & $3.36 \times 10^{3}$ & 0.99 \\
$\mathrm{H}_{2} \mathrm{O}$ & $1.92 \times 10^{-6}$ & 954 & 941 & 0.98 & $7.18 \times 10^{1}$ & 1.04 \\
$\mathrm{CO}_{2}$ & $5.43 \times 10^{-8}$ & 915 & 891 & 1.39 & $1.04 \times 10^{0}$ & 1.40 \\
$\mathrm{CO}^{2}$ & $5.92 \times 10^{-4}$ & 928 & 915 & 1.21 & $1.64 \times 10^{4}$ & 1.20 \\
\hline
\end{tabular}

\subsection{Global view}

We first present a global view on the differences between Model A and Model B. Figure 10 shows the ratio, at an altitude of $1000 \mathrm{~km}$, of the mole fraction obtained with the Model B over the one of the Model A for the most abundant species (with mole fractions greater than $10^{-12}$ in the Model B). For clarity, only a few points are labeled in the figure, those corresponding to the extreme values of the ratio. The most important result is that most of the species have a ratio significantly greater than 1 , meaning that Model A gives abundances notably smaller than model B. Many species have a ratio greater than 2 (a factor of 3.2 for $\mathrm{HCN}$ and 3.4 for $\mathrm{C}_{2} \mathrm{H}_{3} \mathrm{CN}$ for instance) and for several species the ratio exceeds a factor of 5 (a factor of 6.7 for $\mathrm{C}_{2} \mathrm{~N}_{2}$ and 13.6 for $\mathrm{C}_{4} \mathrm{~N}_{2}$ for instance). In particular, among the species that have densities significantly enhanced, many of them are nitrogen species.

We see also that in general, the lower the relative abundance of species, the greater the number of species that show a strong disagreement between the two models. This trend is limited however by the fact that the chemical scheme is far from being complete for heavy species. Indeed, due to the lack of available information in the literature, the number of reactions in our chemical scheme is not proportional to the mass of species, as it should theoretically be (see for instance Fig. 4 in Dobrijevic and Dutour (2007)).

In the lower atmosphere, below $800 \mathrm{~km}$, abundances of ions are too low to give relevant ratios. At $500 \mathrm{~km}$, nitrogen species densities are the ones that are the most enhanced in Model B. The effect of $2 \mathrm{D}$ geometry on the calculation on photolysis rates has no noticeable effect on many hydrocarbons, in 
particular $\mathrm{C}_{2} \mathrm{H}_{2}$ and $\mathrm{C}_{2} \mathrm{H}_{6}$. As observed at an altitude of $1000 \mathrm{~km}$, a general trend can be inferred at $500 \mathrm{~km}$, the heavier the species, the greater the impact of $2 \mathrm{D}$ geometry on its abundance. This behavior might be connected to the increasing coupling between more and more species as the mass of species increases. This is the same behavior we observed when studying the propagation of uncertainties in photochemical models (see for instance Hébrard et al. (2009)).

\subsection{Focus on some species}

We have seen in the previous section that nitriles are the species that present the larger enhancement in their densities at two specific altitudes. We now present the model fraction profiles of several species to point out how large this enhancement as a function of altitude is and to compare the results of Model B with various recent observations, especially on nitriles (see Figures $11,12,13$ and 14).

\subsubsection{Neutral species}

- For HCN (Figure 11), the enhancement factor from Model A to Model B is greater than 2 in the major part of the atmosphere. Model B seems to be in a better agreement with observations from Cassini/CIRS (Vinatier et al. (2010)) and Cassini/INMS (Magee et al. (2009)) than Model A.

- For $\mathrm{HC}_{3} \mathrm{~N}$ (Figure 11), the enhancement factor is equal to or greater than 3 from the ionosphere to the stratosphere with a factor of 4 around $400 \mathrm{~km}$. Model B is in slightly better agreement with both Cassini/CIRS (Vinatier et al. (2010)) and ALMA observations (Cordiner et al. (2019)), but also with INMS data from Cui et al. (2009), which is about 20 times greater than the value inferred by Magee et al. (2009).

- For $\mathrm{C}_{2} \mathrm{~N}_{2}$ (Figure 11), the enhancement factor is relatively high with a value between 5 and 6 from the top of the atmosphere down to $200 \mathrm{~km}$. Model B seems to be in better agreement with Cassini/CIRS observations (Sylvestre et al. (2020)) and in good agreement with Cassini/INMS data retrieved by Magee et al. (2009) but not with Cui et al. (2009).

- For $\mathrm{C}_{4} \mathrm{~N}_{2}$ (Figure 11), the enhancement factor reaches a factor 10 in the mesosphere and stratosphere. There is no direct detection of this species for the moment.

- We obtain also an enhancement factor greater than 2 for $\mathrm{C}_{2} \mathrm{H}_{3} \mathrm{CN}$ and $\mathrm{C}_{2} \mathrm{H}_{5} \mathrm{CN}$. The mole fraction profiles of $\mathrm{C}_{2} \mathrm{H}_{3} \mathrm{CN}$ and $\mathrm{C}_{2} \mathrm{H}_{5} \mathrm{CN}$ derived from Model B seem to be in better agreement with ALMA observation (Cordiner et al. (2019)) but the abundances are still too low at all altitudes. Contribution of the limbs in ALMA data and the increase of nitrile mole fractions 

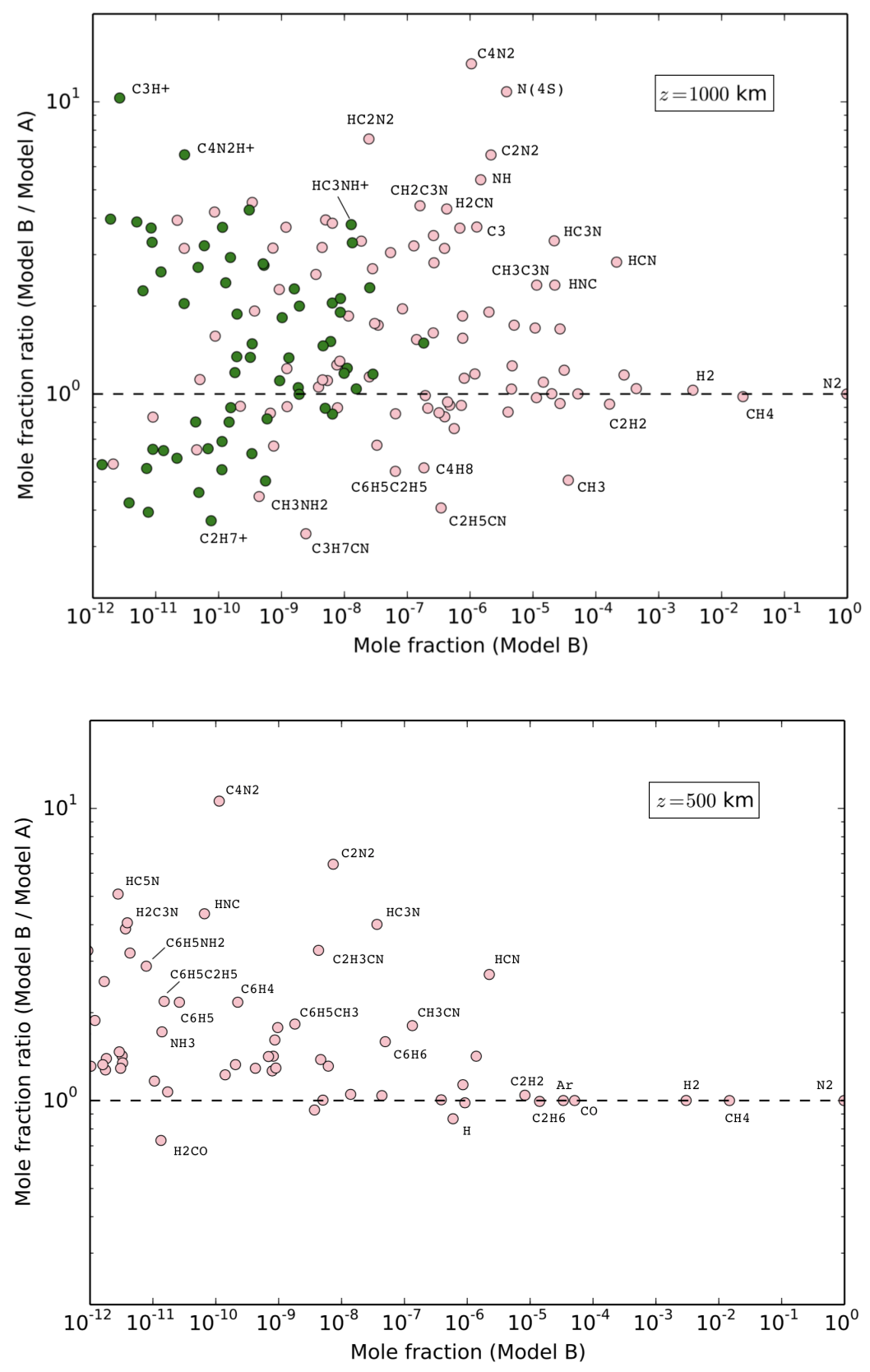

Fig. 10. Ratios of the mole fractions obtained at a given altitude of Model B over Model A. Each point corresponds to a species in the models. Green and pink dots correspond to ions and neutrals respectively. Top: Altitude $z$ equal to $1000 \mathrm{~km}$. Some points with high ratio are labeled. Bottom: Altitude $z$ equal to $500 \mathrm{~km}$. Some particular dots are labeled with the name of the corresponding molecule.

in polar region might contribute to this discrepancy.

- The two isomers of $\mathrm{C}_{3} \mathrm{H}_{4}$ (Figure 12) have been detected recently by the Texas Echelle Cross Echelle Spectrograph on the NASA Infrared Telescope Facility (TEXES), i.e. propadiene (or allene, $\mathrm{CH}_{2} \mathrm{CCH}_{2}$ ) and propyne (or methylacetylene, $\mathrm{CH}_{3} \mathrm{C}_{2} \mathrm{H}$ ), see Lombardo et al. (2019). There is no signifi- 

agreement with observations.

- Using ALMA, Thelen et al. (2020) obtained the first spectroscopic detection of $\mathrm{CH}_{3} \mathrm{C}_{3} \mathrm{~N}$ (methylcyanoacetylene or cyanopropyne) in Titan's atmosphere (Figure 12). It might be interesting to test our profile with a radiative transfert code to better compare our profile with ALMA observations.

- For $\mathrm{c}_{-} \mathrm{C}_{3} \mathrm{H}_{2}$, there is no significant difference between Model $\mathrm{A}$ and Model B. The mole fraction ratio reaches a factor of about 2 in an altitudinal region where the mole fractions are very low. It might be also required to use a radiative transfert code to better compare our profile with ALMA observations.

- $\mathrm{CH}_{3} \mathrm{CN}$ (methyl cyanide) has been also detected by ALMA (see Thelen et al. (2019) and references therein). There is a factor of about 2 between Model A and Model B (Figure 12). Observations seem to slightly favor Model A.

\subsubsection{Ions}

An important constraint for ionospheric models is the electron density. Figure 13 shows the electron density profiles obtained with Model A and Model B and a comparison with observations inferred from Shebanits et al. (2013) based on data from 47 flybys of the Cassini spacecraft Radio and Plasma Wave Science Langmuir Probe (RPWS-LP). Model B is in good agreement with the observed density value at the peak around $1000 \mathrm{~km}$ of altitude. Higher in the ionosphere, the difference between the two models is not significant. According to our results, Model B predicts a much higher electron density in the lower ionosphere (below $800 \mathrm{~km}$ ).

As we can see in Figure 10, many ions have densities significantly different in Model B compared to Model A. We present in Figure 14 the density profiles of six of them with opposite behaviors. In the case of $\mathrm{HCNH}^{+}, \mathrm{HC}_{3} \mathrm{NH}^{+}, \mathrm{NH}_{2}^{+}$ and $\mathrm{CH}_{2} \mathrm{NH}_{2}^{+}$the density is enhanced in Model B compared to Model A. In the case of $\mathrm{C}_{2} \mathrm{H}_{7}^{+}$and $\mathrm{C}_{2} \mathrm{H}_{5} \mathrm{CNH}^{+}$, the density is lower in Model $\mathrm{B}$ in the ionosphere (above the peak of density). Below about $800 \mathrm{~km}$ of altitude, the density in Model B becomes much higher. For the moment, comparison with observations is not conclusive and cannot favor either model. For $\mathrm{HC}_{3} \mathrm{NH}^{+}$, there is a very good agreement between Model B and Cassini/INMS data, while for $\mathrm{NH}_{2}^{+}$, Model A seems to be in better agreement. These differences reflect in part uncertainties in the chemical rates (see Dobrijevic et al. (2016)) and for an another part to incomplete description of the chemical scheme (important reaction may be missing or products poorly known).

In our methodology presented in Figure 3, the next step (step \#3) is to compute the photolysis rates from the atmospheric composition derived from Model B (step \#2), i.e. the density profiles of Model B we used in the present 

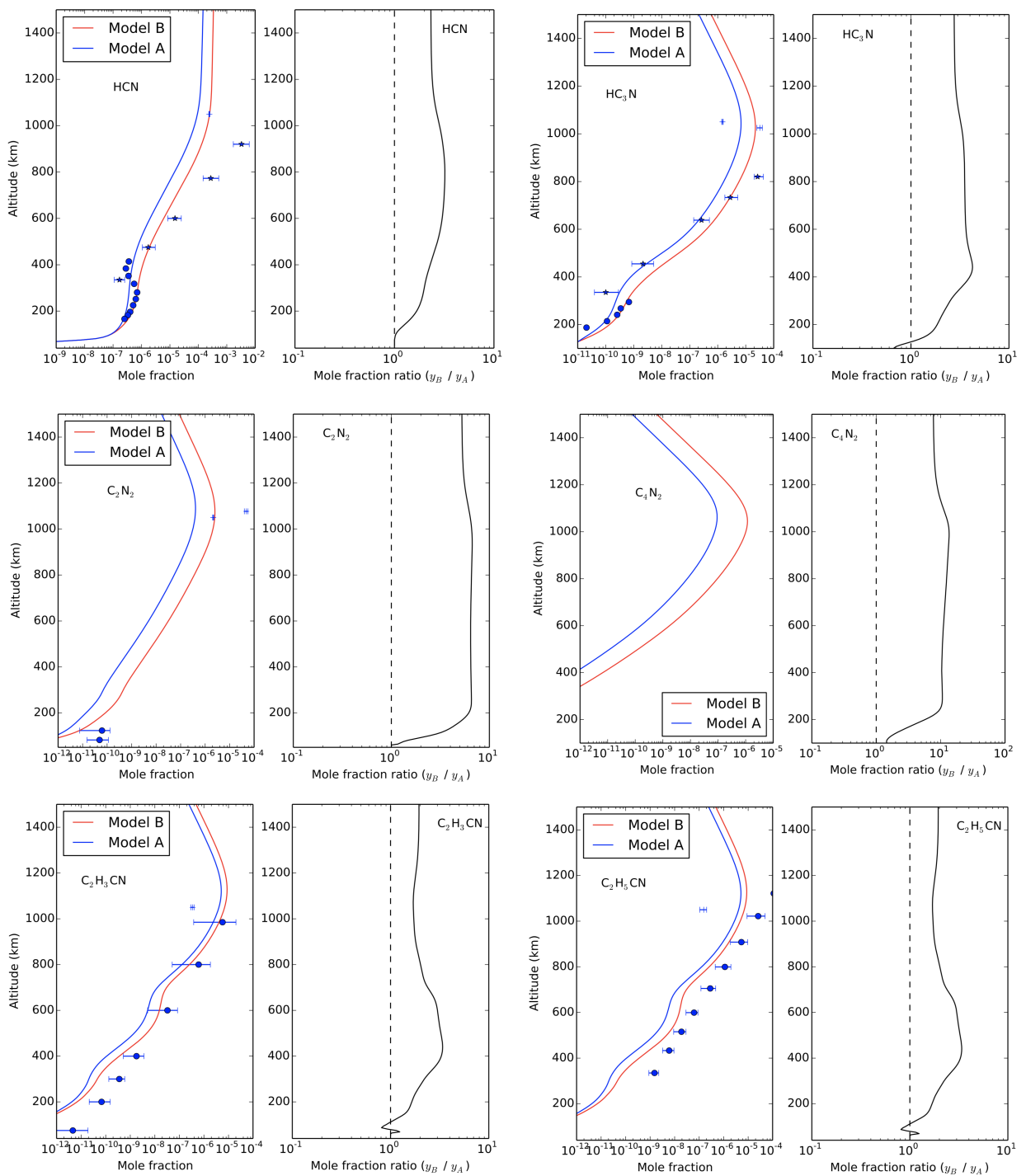

Fig. 11. Mole fraction profiles $y_{i}$ of $\mathrm{HCN}, \mathrm{HC}_{3} \mathrm{~N}, \mathrm{C}_{2} \mathrm{~N}_{2}, \mathrm{C}_{4} \mathrm{~N}_{2}, \mathrm{C}_{2} \mathrm{H}_{3} \mathrm{CN}$ and $\mathrm{C}_{2} \mathrm{H}_{5} \mathrm{CN}$ for the two models $\mathrm{A}$ and $\mathrm{B}$ and their ratio $y_{i}(\mathrm{~B}) / y_{i}(\mathrm{~A})$. Observations come from Cassini/CIRS (HCN and $\mathrm{HC}_{3} \mathrm{~N}$ from Vinatier et al. (2010) and $\mathrm{C}_{2} \mathrm{~N}_{2}$ from Sylvestre et al. (2020)), Cassini/INMS (Magee et al., 2009; Cui et al., 2009) and ALMA (HCN, $\mathrm{HC}_{3} \mathrm{~N}$ and $\mathrm{C}_{2} \mathrm{H}_{5} \mathrm{CN}$ from Cordiner et al. (2019) and $\mathrm{C}_{2} \mathrm{H}_{3} \mathrm{CN}$ from Palmer et al. (2017)).

study. At step \#3, we obtained density profiles similar to the ones obtained at step \#2 meaning that both photolysis rates and density profiles were already at the steady state.

The next step, after step \#3, will be to develop a pseudo-2D model including rotation, in which density profiles will depend also on section (i.e. latitude). Such a model has been developed by De La Haye et al. (2008) using a simple chemical scheme coupling ion and neutral species. They showed that many species, especially ions, have chemical life time much shorter than the rotation 

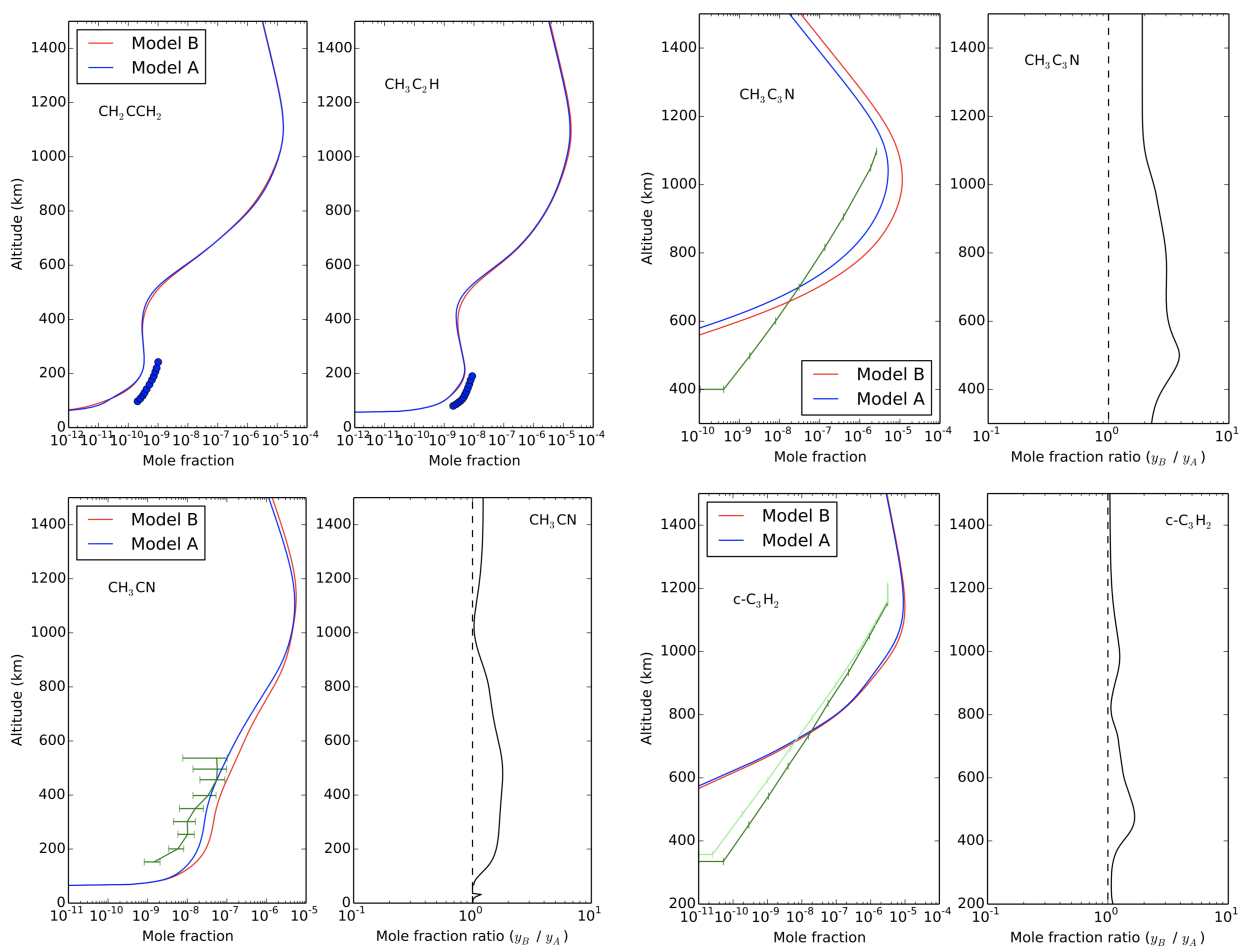

Fig. 12. Mole fraction profiles $y_{i}$ of two isomers of $\mathrm{C}_{3} \mathrm{H}_{4}\left(\mathrm{CH}_{2} \mathrm{CCH}_{2}\right.$ and $\left.\mathrm{CH}_{3} \mathrm{C}_{2} \mathrm{H}\right)$, $\mathrm{CH}_{3} \mathrm{C}_{3} \mathrm{~N}, \mathrm{CH}_{3} \mathrm{CN}$ and $\mathrm{c}-\mathrm{C}_{3} \mathrm{H}_{2}$ for the two models $\mathrm{A}$ and $\mathrm{B}$ and their ratio $y_{i}(\mathrm{~B}) /$ $y_{i}(\mathrm{~A})$ (except for $\mathrm{C}_{3} \mathrm{H}_{4}$ ). Observations come from TEXES (Lombardo et al. (2019) for $\left.\mathrm{C}_{3} \mathrm{H}_{4}\right)$ and ALMA $\left(\mathrm{CH}_{3} \mathrm{C}_{3} \mathrm{~N}\right.$ from Thelen et al. (2020), $\mathrm{CH}_{3} \mathrm{CN}$ from Thelen et al. (2019) and $\mathrm{c}-\mathrm{C}_{3} \mathrm{H}_{2}$ from Nixon et al. (2020)). For c- $\mathrm{C}_{3} \mathrm{H}_{2}$, observations gathered in two different spectral bands are shown.
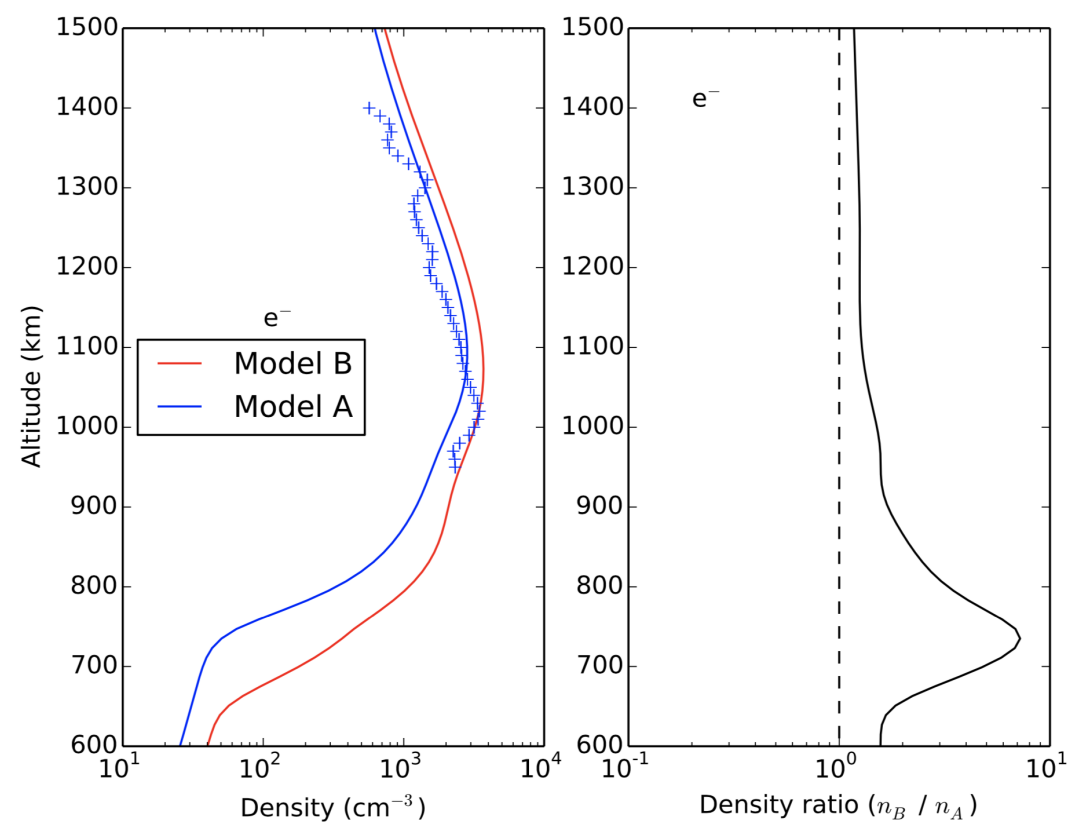

Fig. 13. Density profile of electrons for Model A and Model B. 

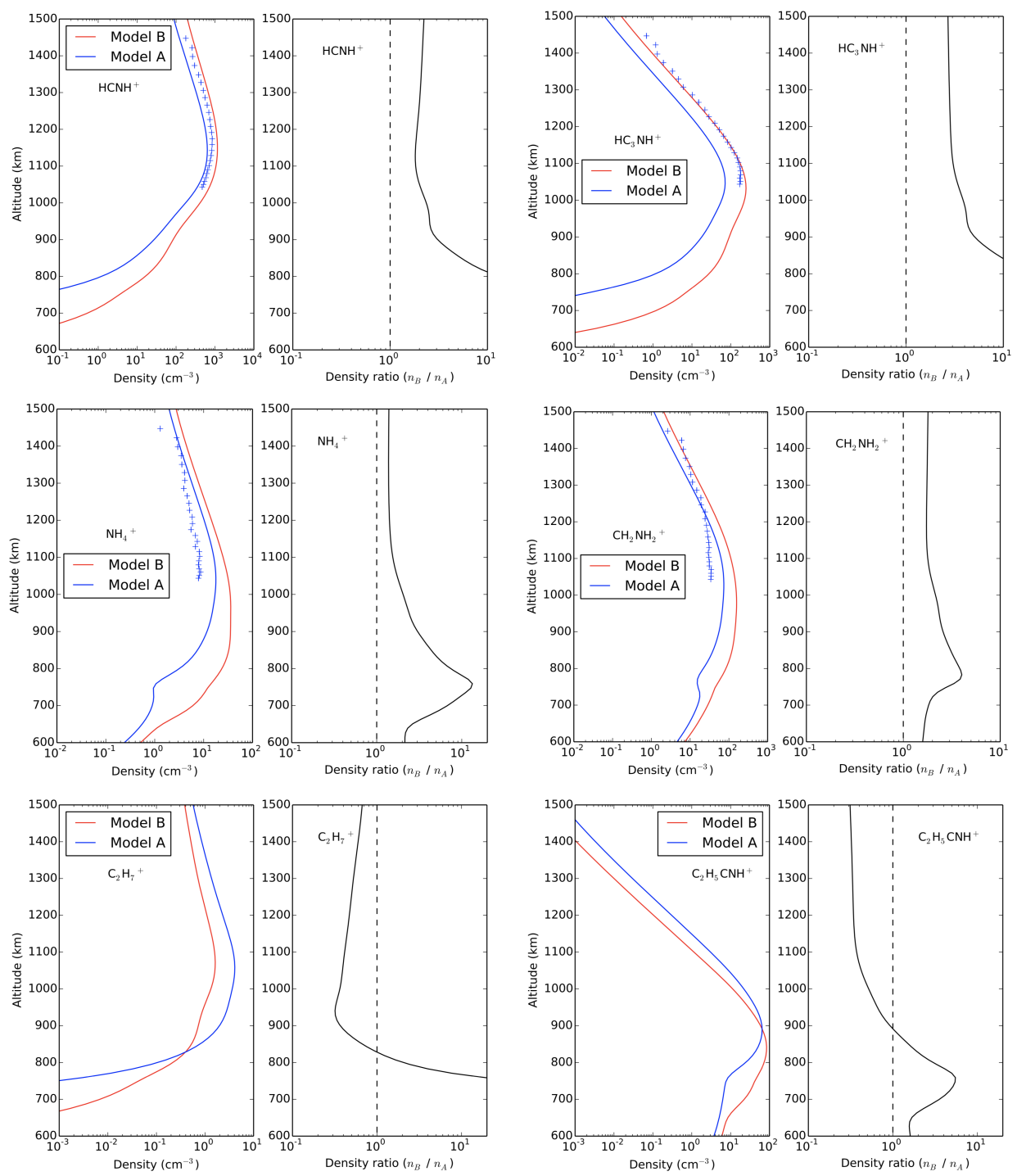

Fig. 14. Densitiy profiles $n_{i}$ of $\mathrm{HCNH}^{+}, \mathrm{HC}_{3} \mathrm{NH}^{+}, \mathrm{NH}_{2}^{+}, \mathrm{CH}_{2} \mathrm{NH}_{4}^{+}, \mathrm{C}_{2} \mathrm{H}_{7}^{+}$and $\mathrm{C}_{2} \mathrm{H}_{5} \mathrm{CNH}^{+}$for the two models $\mathrm{A}$ and $\mathrm{B}$ and their ratio $n_{i}(\mathrm{~B}) / n_{i}(\mathrm{~A})$. Observations come from Cassini/INMS (Westlake et al., 2012).

period of Titan. Ions and other species (like $\mathrm{HCN}$ ) exhibit strong local timedependence. However, they used a rotating technique at constant latitude and varying local-time to account for the diurnal variation of solar inputs. We intend to improve their model using calculation of actinic flux in 2D geometry. Such an extension deserves a dedicated study beyond the scope of the present paper. 


\section{Conclusion}

We made a comparison between two types of 1D photochemical models used in mean conditions. In the first model (Model A), the photolysis rates are computed by a classic plane-parallel ultraviolet radiative transfer model. In the second model (Model B), the ultraviolet radiative transfer model is based on a $2 \mathrm{D}$ geometry. We first compared the mean photolysis rates for each photolysis process derived by the two models and found the following results:

- The mean photodissociation rates obtained with a 2D geometry model are generally greater than those obtained in a plane-parallel model. For many species, the enhancement factor is greater than 1.2. For some species, the enhancement factor reaches 1.4. For photoionisation, the results are more disparate.

- Considering the various branching paths of a given photodissociation rate, we found that the relative rates of each path can be affected differently, when the 2D geometry is accounted for photolysis, changing the relative importance of each path in Model B compared to Model A.

- We also obtained that the location of the photolysis peaks are generally slightly lower in altitude.

All these characteristics sufficiently affect the photochemical processes to give fraction profiles significantly different in Model B compared to Model A.

- We found little differences for some light species between the two models, in particular for light hydrocarbons like $\mathrm{C}_{2} \mathrm{H}_{2}, \mathrm{C}_{2} \mathrm{H}_{4}$ and $\mathrm{C}_{2} \mathrm{H}_{6}$. This, in fact, validates the use of $1 \mathrm{D}$ model in mean conditions for these species compared to a model using 2D geometry for calculating the actinic flux.

- The differences are more marked for many heavier species and especially for nitriles (from C3-hydrocarbons and other nitrogen species, i.e. the ones observers will target with the next generation of observatories). This effect is due to the fact that heavier species depend on several lighter species.

- Many density profiles of ions calculated from Model B are significantly different from the ones obtained with Model A. This concerns major and minor species as well.

Note that, for most of the species, uncertainties on model results are important and do not permit to distinguish Model A and Model B profiles for the moment (see Dobrijevic et al. (2016)). Pending a decrease of these uncertainties (as we obtained for light hydrocarbons improving the chemical scheme over the past few years from Hébrard et al. (2007) to Dobrijevic et al. (2016)), it remains that the general tendency is that we obtain an increase in photochemical products from Model A to Model B. 
Thus, we showed that a 1D photochemical model in which mean photolysis rates are calculated from actinic fluxes derived in 2D geometry gives results significantly different from a classical 1D photochemical model in mean conditions. We then advocate the use of $2 \mathrm{D}$ geometry from now on to compute the actinic flux and mean photolysis rates.

Additionally, we consider that this procedure is an interesting step to fill the gap between 1D models with large chemical scheme but limited physics (radiative balance, dynamics, cloud microphysics, etc.) and GCM-type models with very simplified chemical schemes (if any). This allows the development of $2 \mathrm{D}$ models allowing a better assessment of the effect of photolysis, the day-night average and putative latitudinal variations of the fluxes of in-falling species. Such 2D models should be a better tool than 1D models to interpret the numerous data gathered by the Cassini space probe with the possibility to better simulate variations with latitude. It would be also a better model to interpret disk-averaged observations or low spatial resolution observations obtained from the ground with a global mean approximation.

\section{Acknowledgements}

We are grateful for the constructive reviews that have significantly improved the manuscript. 


\section{References}

Cordiner, M. A., Palmer, M. Y., Nixon, C. A., Irwin, P. G. J., Teanby, N. A., Charnley, S. B., Mumma, M. J., Kisiel, Z., Serigano, J., Kuan, Y.-J., Chuang, Y.-L., Wang, K.-S., Feb. 2015. Ethyl Cyanide On Titan: Spectroscopic Detection and Mapping Using Alma. The Astrophysical Journal Letters 800, L14.

Cordiner, M. A., Teanby, N. A., Nixon, C. A., Vuitton, V., Thelen, A. E., Charnley, S. B., Aug. 2019. ALMA Spectral Imaging of Titan Contemporaneous with Cassini's Grand Finale. The Astronomical Journal 158 (2), 76.

Cui, J., Yelle, R. V., Vuitton, V., Waite, J. H., Kasprzak, W. T., Gell, D. A., Niemann, H. B., Müller-Wodarg, I. C. F., Borggren, N., Fletcher, G. G., Patrick, E. L., Raaen, E., Magee, B. A., Apr. 2009. Analysis of Titan's neutral upper atmosphere from Cassini Ion Neutral Mass Spectrometer measurements. Icarus 200, 581-615.

De La Haye, V., Waite, J. H., Cravens, T. E., Robertson, I. P., Lebonnois, S., Sep. 2008. Coupled ion and neutral rotating model of Titan's upper atmosphere. Icarus 197 (1), 110-136.

Dobrijevic, M., Dutour, I., Nov. 2007. The distribution of hydrocarbons in Titan's atmosphere: An evolutionary algorithm-based model. Planetary and Space Science 55 (14), 2128-2136.

Dobrijevic, M., Hébrard, E., Loison, J. C., Hickson, K. M., Jan. 2014. Coupling of oxygen, nitrogen, and hydrocarbon species in the photochemistry of Titan's atmosphere. Icarus 228, 324-346.

Dobrijevic, M., Loison, J. C., Jun 2018. The photochemical fractionation of nitrogen isotopologues in Titan's atmosphere. Icarus 307, 371-379.

Dobrijevic, M., Loison, J. C., Hickson, K. M., Gronoff, G., 2016. 1D-coupled photochemical model of neutrals, cations and anions in the atmosphere of Titan. Icarus 268, 313-339.

Hartogh, P., Lellouch, E., Moreno, R., Bockelée-Morvan, D., Biver, N., Cassidy, T., Rengel, M., Jarchow, C., Cavalié, T., Crovisier, J., Helmich, F. P., Kidger, M., Aug. 2011. Direct detection of the Enceladus water torus with Herschel. Astronomy and Astrophysics 532, L2.

Hébrard, E., Dobrijevic, M., Bénilan, Y., Raulin, F., 2007. Photochemical kinetics uncertainties in modeling Titan's atmosphere: First consequences. Planetary and Space Science 55, 1470-1489.

Hébrard, E., Dobrijevic, M., Pernot, P., Carrasco, N., Bergeat, A., Hickson, K. M., Canosa, A., Le Picard, S. D., Sims, I. R., 2009. How Measurements of Rate Coefficients at Low Temperature Increase the Predictivity of Photochemical Models of Titan's Atmosphere. Journal of Physical Chemistry 113 (42), 11227-11237.

Hickson, K. M., Loison, J. C., Cavalié, T., Hébrard, E., Dobrijevic, M., Dec. 2014. The evolution of infalling sulfur species in Titan's atmosphere. Astronomy and Astrophysics 572, A58. 
Hörst, S. M., Vuitton, V., Yelle, R. V., Oct. 2008. Origin of oxygen species in Titan's atmosphere. Journal of Geophysical Research (Planets) 113, 10006.

Krasnopolsky, V. A., May 2009. A photochemical model of Titan's atmosphere and ionosphere. Icarus 201, 226-256.

Krasnopolsky, V. A., Jul. 2014. Chemical composition of Titan's atmosphere and ionosphere: Observations and the photochemical model. Icarus 236, 8391.

Krasnopolsky, V. A., Dec 2016. Isotopic ratio of nitrogen on Titan: Photochemical interpretation. Planetary and Space Science 134, 61-63.

Lara, L. M., Lellouch, E., González, M., Moreno, R., Rengel, M., Jun. 2014. A time-dependent photochemical model for Titan's atmosphere and the origin of $\mathrm{H}_{2} \mathrm{O}$. Astronomy and Astrophysics 566, A143.

Liang, M.-C., Heays, A. N., Lewis, B. R., Gibson, S. T., Yung, Y. L., Aug 2007. Source of Nitrogen Isotope Anomaly in HCN in the Atmosphere of Titan. The Astrophysical Journal 664 (2), L115-L118.

Loison, J. C., Dobrijevic, M., Hickson, K. M., Sep 2019. The photochemical production of aromatics in the atmosphere of Titan. Icarus 329, 55-71.

Loison, J. C., Dobrijevic, M., Hickson, K. M., Heays, A. N., Jul. 2017. The photochemical fractionation of oxygen isotopologues in Titan's atmosphere. Icarus 291, 17-30.

Loison, J. C., Hébrard, E., Dobrijevic, M., Hickson, K. M., Caralp, F., Hue, V., Gronoff, G., Venot, O., Bénilan, Y., 2015. The neutral photochemistry of nitriles, amines and imines in the atmosphere of Titan. Icarus 247, 218-247.

Lombardo, N. A., Nixon, C. A., Greathouse, T. K., Bézard, B., Jolly, A., Vinatier, S., Teanby, N. A., Richter, M. J., Irwin, P. J. G., Coustenis, A., Flasar, F. M., Aug 2019. Detection of Propadiene on Titan. The Astrophysical Journal Letters 881 (2), L33.

Magee, B. A., Waite, J. H., Mandt, K. E., Westlake, J., Bell, J., Gell, D. A., Dec. 2009. INMS-derived composition of Titan's upper atmosphere: Analysis methods and model comparison. Planet. Space Sci. 57, 1895-1916.

Mandt, K. E., Waite, J. H., Lewis, W., Magee, B., Bell, J., Lunine, J., Mousis, O., Cordier, D., Dec. 2009. Isotopic evolution of the major constituents of Titan's atmosphere based on Cassini data. Planetary and Space Science 57 (14-15), 1917-1930.

Moreno, R., Lellouch, E., Lara, L. M., Feuchtgruber, H., Rengel, M., Hartogh, P., Courtin, R., Nov. 2012. The abundance, vertical distribution and origin of $\mathrm{H}_{2} \mathrm{O}$ in Titan's atmosphere: Herschel observations and photochemical modelling. Icarus 221, 753-767.

Moses, J. I., Poppe, A. R., Nov. 2017. Dust ablation on the giant planets: Consequences for stratospheric photochemistry. Icarus 297, 33-58.

Mukundan, V., Bhardwaj, A., apr 2018. A model for negative ion chemistry in titan's ionosphere. The Astrophysical Journal 856 (2), 168.

Nixon, C. A., Thelen, A. E., Cordiner, M. A., Kisiel, Z., Charnley, S. B., Molter, E. M., Serigano, J., Irwin, P. G. J., Teanby, N. A., Kuan, Y.-J., Nov. 2020. Detection of Cyclopropenylidene on Titan with ALMA. The 
Astronomical Journal 160 (5), 205.

Nunez-Reyes, D., Loison, J.-C., Hickson, K. M., Dobrijevic, M., 2019a. A low temperature investigation of the $\mathrm{n}(2 \mathrm{~d})+\mathrm{ch} 4, \mathrm{c} 2 \mathrm{~h} 6$ and $\mathrm{c} 3 \mathrm{~h} 8$ reactions. Physical Chemistry Chemical Physics 21 (12), 6574-6581. URL http://dx.doi.org/10.1039/C9CP00798A

Nunez-Reyes, D., Loison, J.-C., Hickson, K. M., Dobrijevic, M., 2019b. Rate constants for the $\mathrm{n}(2 \mathrm{~d})+\mathrm{c} 2 \mathrm{~h} 2$ reaction over the $50296 \mathrm{k}$ temperature range. Physical Chemistry Chemical Physics. URL http://dx.doi.org/10.1039/C9CP04170B

Palmer, M. Y., Cordiner, M. A., Nixon, C. A., Charnley, S. B., Teanby, N. A., Kisiel, Z., Irwin, P. G. J., Mumma, M. J., Jul 2017. ALMA detection and astrobiological potential of vinyl cyanide on Titan. Science Advances 3 (7), e1700022.

Shebanits, O., Wahlund, J.-E., Mandt, K., Ågren, K., Edberg, N. J. T., Waite, J. H., Aug. 2013. Negative ion densities in the ionosphere of Titan-Cassini RPWS/LP results. Planetary and Space Science 84, 153-162.

Sylvestre, M., Teanby, N. A., Dobrijevic, M., Sharkey, J., Irwin, P. G. J., Oct. 2020. $\mathrm{C}_{2} \mathrm{~N}_{2}$ Vertical Profile in Titan's Stratosphere. The Astronomical Journal 160 (4), 178.

Teanby, N. A., Cordiner, M. A., Nixon, C. A., Irwin, P. G. J., Hörst, S. M., Sylvestre, M., Serigano, J., Thelen, A. E., Richards, A. M. S., Charnley, S. B., Jun 2018. The Origin of Titan's External Oxygen: Further Constraints from ALMA Upper Limits on $\mathrm{CS}$ and $\mathrm{CH}_{2} \mathrm{NH}$. The Astronomical Journal 155 (6), 251.

Thelen, A. E., Cordiner, M. A., Nixon, C. A., Vuitton, V., Kisiel, Z., Charnley, S. B., Palmer, M. Y., Teanby, N. A., Irwin, P. G. J., Nov. 2020. Detection of $\mathrm{CH}_{3} \mathrm{C}_{3} \mathrm{~N}$ in Titan's Atmosphere. Astrophysical Journal Letters 903 (1), L22.

Thelen, A. E., Nixon, C. A., Chanover, N. J., Cordiner, M. A., Molter, E. M., Teanby, N. A., Irwin, P. G. J., Serigano, J., Charnley, S. B., Feb. 2019. Abundance measurements of Titan's stratospheric $\mathrm{HCN}, \mathrm{HC}_{3} \mathrm{~N}, \mathrm{C}_{3} \mathrm{H}_{4}$, and $\mathrm{CH}_{3} \mathrm{CN}$ from ALMA observations. Icarus 319, 417-432.

Vinatier, S., Bézard, B., Lebonnois, S., Teanby, N. A., Achterberg, R. K., Gorius, N., Mamoutkine, A., Guandique, E., Jolly, A., Jennings, D. E., Flasar, F. M., Apr. 2015. Seasonal variations in Titan's middle atmosphere during the northern spring derived from Cassini/CIRS observations. Icarus 250, 95-115.

Vinatier, S., Bézard, B., Nixon, C. A., Mamoutkine, A., Carlson, R. C., Jennings, D. E., Guandique, E. A., Teanby, N. A., Bjoraker, G. L., Michael Flasar, F., Kunde, V. G., Feb. 2010. Analysis of Cassini/CIRS limb spectra of Titan acquired during the nominal mission. I. Hydrocarbons, nitriles and $\mathrm{CO}_{2}$ vertical mixing ratio profiles. Icarus 205 (2), 559-570.

Vuitton, V., Yelle, R. V., Klippenstein, S. J., Hörst, S. M., Lavvas, P., May 2019. Simulating the density of organic species in the atmosphere of Titan with a coupled ion-neutral photochemical model. Icarus 324, 120-197. 
706 Westlake, J. H., Waite, J. H., J., Mandt, K. E., Carrasco, N., Bell, J. M., 707 Magee, B. A., Wahlund, J. E., Jan. 2012. Titan's ionospheric composition 708 and structure: Photochemical modeling of Cassini INMS data. Journal of 709 Geophysical Research (Planets) 117 (E1), E01003. 